International Journal of Robotics and Automation (IJRA)

Vol. 10, No. 3, September 2021, pp. 235 260

ISSN: 2089-4856, DOI: 10.11591/ijra.v10i3.pp235-260

\title{
Control of teleoperation systems in the presence of cyber attacks: A survey
}

\author{
Mutaz M. Hamdan, Magdi S. Mahmoud \\ Systems Engineering Department, King Fahd University of Petroleum \& Minerals, Saudi Arabia
}

\begin{abstract}
Article Info
Article history:

Received May 28, 2021

Revised Jul 9, 2021

Accepted Jul 16, 2021

\section{Keywords:}

Bilateral teleoperation system

ABSTRACT

The teleoperation system is often composed of a human operator, a local master manipulator, and a remote slave manipulator that are connected by a communication network. This paper proposes a survey on feedback control design for the bilateral teleoperation systems (BTSs) in nominal situations and in the presence of cyber-attacks. The main idea of the presented methods is to achieve the stability of a delayed bilateral teleoperation system in the presence of several kinds of cyber attacks. In this paper, a comprehensive survey on control systems for BTSs under cyber-attacks is discussed. Finally, we discuss the current and future problems in this field.
\end{abstract} Cyber attack

Denial of service (DoS) attack

Passivity based control

Telesurgical robot systems

Wave variable-based control
This is an open access article under the CC BY-SA license.

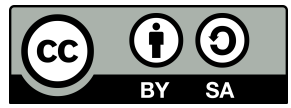

\section{Corresponding Author:}

Magdi S. Mahmoud

Systems Engineering Department

King Fahd University of Petroleum Minerals

P. O. Box 5067, Dhahran 31261, Saudi Arabia

Email: msmahmoud@kfupm.edu.sa

\section{INTRODUCTION}

A teleoperation system is referred to a plant that is controlled remotely. Many teleoperation systems have been designed and used in the recent decades to help people in performing tasks remotely especially in hazardous environments. These tasks include working in a toxic or harmful environment, working remotely like telesurgery and explorations of space, and carrying out high precision tasks such as chemical and nuclear reactors. In general, teleoperation systems consist of a human operator, a master and slave manipulators connected through a communication network and the environment. The schematic diagram of a typical teleoperation system is shown in Figure 1.

Teleoperation systems allow operator to exchange information of position, velocity, and/or force remotely to perform the desired motion, sensing, and physical manipulation. The importance and wide applications of teleoperation systems attract researchers in both control systems and robotics mainly focusing on the stability and the telepresence [1]-[3]. A lot of result are presented to handle practical control problems using several approaches such as anti-wind control [4], [5], fuzzy control [6], [7], adaptive control [8]-[13], passivitybased control [2], [14], [15], and slide-mode control [16], [17]. We will discuss these and other approaches in details in section 3 .

There are two main types of teleoperation systems: unilateral and bilateral teleoperation systems. When the transmission of motion goes from master to slave it is called a unilateral teleoperation system (UTS). On the other hand, a bilateral teleoperation system (BTS) includes transmissions in both the forward and backward directions between the master and slave [18]. Generally speaking, BTSs have more interest among researchers since they are more used in places that are difficult for humans such as underwater vehicles, airspace 
applications, mining, and remote medical surgeries. Comprehensive historical surveys on BTSs are presented in [1], [19]. In 2011, Nuño, Basañez and Ortega [2], a tutorial on passivity based control on BTSs is provided. A survey on the environment and task-based controller system is shown in [20]. Also, a review on bilateral teleoperation with force, position, power, and impedance scaling is detailed in [21].

In addition to the basic requirements of stability, a major aspect to consider in BTSs is the transparency. Actually, there is a tradeoff between these two concerns mainly as a result of the time delays caused by the communication channel [22]. The delay-induced instability of BTSs is one of the main challenges for researchers since time delays exist in most communication channels. Some major studies based on timedelayed bilateral teleoperation have been done in [12], [23]-[25]. The time delays could be symmetric [26] as well as asymmetric [27]. Some recent studies on the stability analysis of asymmetric time-varying delay can be found in [28]-[30]. Most of the recent work on stability analysis with asymmetric time-varying delays employed Lyapunov-Krasovskii functional to formulate the relations among the controller parameters, and the upper bound of time-varying delays [31], [32]. Linear matrix inequalities (LMIs) were applied to present the stability criteria. So, it can be solved to obtain the allowed maximum values of delays.

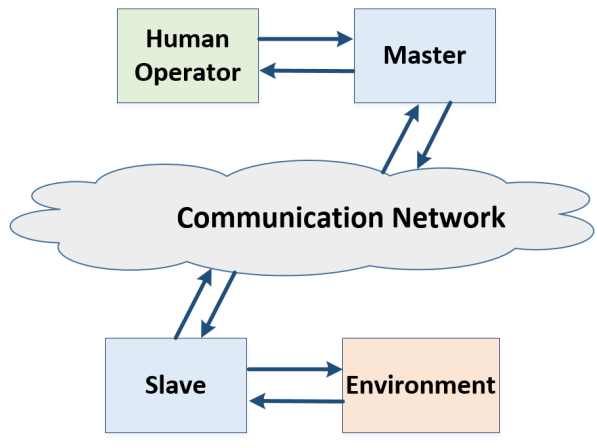

Figure 1. Block diagram of the typical teleoperation system

Recently, many reviews on the BTSs were published. In 2018, Ghavifekr, Ghiasi and Badamchizadeh [33], discrete-time control methods of BTSs were presented with concern on problems of passivity, stability, transparency, and time delays. The recent control approaches for teleoperation systems while considering internet-based communication, unknown time-varying delay, and model uncertainty were reviewed [34]. More concern was given to control algorithms that were applied to nonlinear uncertain systems. The application of predictive control schemes in BTSs are discussed including qualitative and quantitative comparisons among these methods regarding robustness, transparency, and stability [35].

In this paper we address the control problem of bilateral teleoperation systems (BTSs) in nominal situations and in the presence of cyber-attacks. The main contributions of this paper are summarized as follows:

- A discussion on the methods of modeling BTSs will be presented.

- A comprehensive survey on control schemes for BTSs under cyber-attacks is discussed.

- The literature that considers cyber-attacks in the design of the controller for BTSs is reviewed.

- The current and future problems in this field are discussed.

The remaining of this paper is organized as follows: Modeling of BTSs are discussed in section 2. In section 3, control approaches are presented. Then, the cyber-attacks in BTSs are described in section 4. Finally, Current and future research are listed in section 5.

\section{MODELING OF BTSS}

In section 2, we will discuss the modeling of BTSs. The dynamics of both the master and slave robots are derived by applying the Lagrangian systems with the actuated revolute joints such that shown in (1):

$$
\begin{aligned}
& M_{m}\left(q_{m}\right) \ddot{q}_{m}+C_{m}\left(q_{m}, \dot{q}_{m}\right) \dot{q}_{m}+g_{m}\left(q_{m}\right)=J_{m}^{T}\left(q_{m}\right) F_{m}+\tau_{m} \\
& M_{s}\left(q_{s}\right) \ddot{q}_{s}+C_{s}\left(q_{s}, \dot{q}_{s}\right) \dot{q}_{s}+g_{s}\left(q_{s}\right)=-J_{s}^{T}\left(q_{s}\right) F_{s}+\tau_{s}
\end{aligned}
$$


with the subscripts $\{m\}$ and $\{s\}$ denote the master robot and the slave robot, respectively. $q_{m}(t) \in^{n}$ and $q_{s}(t) \in^{m}$ are the generalized coordinates, $M_{m}\left(q_{m}\right) \in^{n \times n}$ and $M_{s}\left(q_{s}\right) \in^{m \times m}$ are the inertia matrices.

$C_{m}\left(q_{m}, \dot{q}_{m}\right) \in^{n \times n}$ and $C_{s}\left(q_{s}, \dot{q}_{s}\right) \in^{m \times m}$ are damping matrices. $g_{m}\left(q_{m}\right) \in^{n}$ and $g_{s}\left(q_{s}\right) \in^{m}$ are the gravity forces. $\tau_{m}(t) \in^{n}$ and $\tau_{s}(t) \in^{m}$ are the applied torques which is deifned sometimes as the control signals. $J_{m}\left(q_{m}\right) \in^{n \times n}$ and $J_{s}\left(q_{s}\right) \in^{n \times m}$ are the Jacobian matrices, and $F_{m}(t), F_{s}(t) \in^{n \times 1}$ are the forces exerted by the human operator and the environment. See for example [10], [36], [37]. Let us assume that the slave robot is a redundant manipulator to obtain semi-autonomous teleoperation and the master robot is non-redundant manipulator for simplicity.

Let $x(t)$ defined as the state vector and equal to $\left[x_{m}(t) \dot{x}_{m}(t) x_{s}(t) \dot{x}_{s}(t)\right]^{T}$ with $x(t)$ and $\dot{x}$ are denoting the position and speed of the end effector, respectively. And by considering the external forces as disturbances, the general state-space model for system (1) is represented by (2):

$$
\dot{x}(t)=A x(t)+B u(t)+B_{w} w(t)
$$

with

$$
\begin{aligned}
& A=\left[\begin{array}{cccc}
0 & 1 & 0 & 0 \\
0 & -\frac{B_{m}}{M_{m}} & 0 & 0 \\
0 & 0 & 0 & 1 \\
0 & 0 & 0 & -\frac{B_{s}}{M_{s}}
\end{array}\right] \\
& B=\left[\begin{array}{cc}
0 & 0 \\
\frac{1}{M_{m}} & 0 \\
0 & 0 \\
0 & \frac{1}{M_{s}}
\end{array}\right]=\left[\begin{array}{ll}
B_{1} & B_{2}
\end{array}\right] \\
& B_{1}=\left[\begin{array}{c}
0 \\
\frac{1}{M_{m}} \\
0 \\
0
\end{array}\right], B_{2}=\left[\begin{array}{c}
0 \\
0 \\
0 \\
\frac{1}{M_{s}}
\end{array}\right], B_{w}=\left[\begin{array}{cc}
0 & 0 \\
\frac{1}{M_{m}} & 0 \\
0 & 0 \\
0 & -\frac{1}{M_{s}}
\end{array}\right] \\
& u(t)=\left[\begin{array}{ll}
F_{m}(t) & F_{s}(t)
\end{array}\right]^{T}, w(t)=\left[\begin{array}{ll}
F_{h}(t) & F_{e}(t)
\end{array}\right]^{T}
\end{aligned}
$$

In equation (2), the state variables are $x_{m}(t), \dot{x}_{m}(t), x_{s}(t)$, and $\dot{x}_{s}(t)$. For simplicity, the states $x_{m}(t)$ and $\dot{x}_{m}(t)$ are called local state variables and $x_{s}(t)$ and $\dot{x}_{s}(t)$ are called remote state variables to the master, and conversely with respect to the slave.

The following state-feedback controllers are designed to obtain the stability for the master and slave manipulators as (4):

$$
\begin{aligned}
F_{m}(t) & =K_{m}\left[\begin{array}{llll}
x_{m}(t) & \dot{x}_{m}(t) & x_{s}\left(t-d_{2}(t)\right) & \dot{x}_{s}\left(t-d_{2}(t)\right)
\end{array}\right]^{T} \\
F_{s}(t) & =K_{s}\left[\begin{array}{lll}
x_{m}\left(t-d_{1}(t)\right) & \dot{x}_{m}\left(t-d_{1}(t)\right) & x_{s}(t) \dot{x}_{s}(t)
\end{array}\right]^{T}
\end{aligned}
$$

where $d_{1}(t)$ and $d_{2}(t)$ are the forward time delay (the communication path from the master to the slave) and the backward time delay (the communication path from the slave to the master), respectively.

Remark 1. It is more practical to have upper limit on the time delays such as positive scalars $\bar{d}_{1}$ and $\bar{d}_{2}$. So, $d_{1}(t)$ and $d_{2}(t)$ are bounded as, $0 \leq d_{1}(t) \leq \bar{d}_{1}, 0 \leq d_{2}(t) \leq \bar{d}_{2}$ [38].

The controllers $F_{m}(t)$ and $F_{s}(t)$ is formulated in the following compact system:

$$
\begin{aligned}
F_{m}(t) & =K_{m} x\left(t-d_{2}(t)\right) \\
F_{s}(t) & =K_{s} x\left(t-d_{1}(t)\right)
\end{aligned}
$$

By substituting the controllers (5) in the BTS (3), one will obtain the following overall closed-loop system shown in (6):

$$
\dot{x}(t)=A x(t)+B_{1} K_{m} x\left(t-d_{2}(t)\right)+B_{2} K_{s} x\left(t-d_{1}(t)\right)+B_{w} w(t)
$$




\section{CONTROL METHODS FOR BTSS}

Many literature discuss the challenges of controlling teleoperation systems [39]. The focus of these surveys directed to adaptive control, wave variable, and predictive control approaches [35], [40], [41]. Various methodologies were proposed to handle control issues in several applications of teleoperation systems. Some examples are: nonlinear trilateral teleoperation systems [42], PD-like controller [43], four-channel structure [44], operator dynamics considerations [45], output feedback with force estimation [46], a stable and transparent microscale teleoperation [47], external force estimation [48], position synchronization [49], and new stability criteria using hybrid strategies of position and impedance reflection [50].

As mentioned in the Introduction, some recent literature discusses the control of BTSs. In 2018, Ghavifekr et al. [33], discrete-time control methods of BTSs were presented with concern on problems of passivity, stability, transparency, and time delays. The recent control approaches for teleoperation systems while considering internet-based communication, unknown time-varying delay, and model uncertainty were reviewed [34]. More concern was given to control algorithms that are applicable to nonlinear uncertain systems. The application of predictive control schemes in BTSs are discussed including qualitative and quantitative comparisons among these methods regarding robustness, transparency, and stability [35]. In section 3, we will discuss several kinds of control approaches applied to BTSs and the feature of each method will be explained. These approaches are summarized in Figure 2.

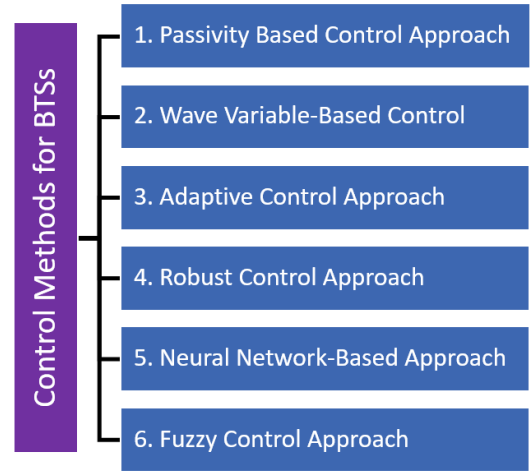

Figure 2. Control approaches applied to BTSs

\subsection{Passivity based control approach}

Passive systems are referred to dynamical systems that have positive consumption of total energy. Passivity based control is a control approach that aims to maintain the passivity behavior of the closed-loop system. So, in this approach, the controller is designed and formulated to keep the balance of the power flow and energy consumption of the BTS [51]. One of the challenges in BTSs is the occurrence of time delays in the communication channel. In addition to its effect on the signal, the time delay could also increase the energy in the communication channel [52]. This could affect the passivity of the BTS and may lead to instability. To handle this issue, several control approaches based on passivity have been proposed. The passivity-based control approach is suitable and applicable to nonlinear systems, distinctly to mechanical/Euler-Lagrange systems [53].

Let us consider the BTS (1), the input power of this BTS in terms of the passivity is given by [54]:

$$
P_{\text {in }}(t)=x^{T} y
$$

where $x$ is the input and $y$ is the output of the system.

The input power flow in the BTS (1) is formulated as (8):

$$
P_{\text {in }}(t)=\left[\begin{array}{ll}
\dot{q}_{m}^{T} & f_{e}^{T}
\end{array}\right]\left[\begin{array}{c}
f_{h} \\
-\dot{q}_{s}
\end{array}\right] .
$$

The passivity of system (1) is assured if and only if a state function $E\left(q_{i}, \dot{q}_{i}\right) \geq 0, \forall\left(q_{i}, \dot{q}_{i}\right)$ exists and satisfies the following inequality [54] as (9):

$$
\frac{d}{d t} E\left(q_{i}, \dot{q}_{i}\right) \leq P_{\text {in }}
$$



[51], [53].

Many literature refers to the energy storage function by $E\left(q_{i}, \dot{q}_{i}\right)$ and the dissipative rate by $\frac{d}{d t} E\left(q_{i}, \dot{q}_{i}\right)$

The previous inequality (9) is represented by the following (10):

$$
\frac{\partial E\left(q_{i}, \dot{q}_{i}\right)}{\partial q_{i}} \dot{q}_{i}+\frac{\partial E\left(q_{i}, \dot{q}_{i}\right)}{\partial \dot{q}_{i}} \ddot{q}_{i} \leq\left[\begin{array}{ll}
\dot{q}_{m}^{T} & f_{e}^{T}
\end{array}\right]\left[\begin{array}{c}
f_{h} \\
\dot{q}_{s}
\end{array}\right]
$$

Now, consider system (1) and several manipulations to describe the torque control signals $\tau_{i}$ as (11):

$$
\frac{\partial E}{\partial \dot{q}_{i}} M_{i}^{-1} \tau_{i} \leq\left[\begin{array}{cc}
\dot{q}_{m}^{T} & f_{e}^{T}
\end{array}\right]\left[\begin{array}{c}
f_{h} \\
\dot{q}_{s}
\end{array}\right]-\frac{\partial E}{\partial q_{i}} \dot{q}_{i}+\frac{\partial E}{\partial \dot{q}_{i}} M_{i}^{-1}\left(C_{i} q_{i}+g_{i}-f_{j}\right)
$$

where $i \in\{m, s\}$ andj $\in\{h, e\}$. Finally, the passivity-based control approach is summarized as follows: Design a suitable $\tau_{i}$ such that (11) holds for all $q_{i}$ and $\dot{q}_{i}$.

Many works of literature investigate this control approach. In E. Kamrani [55], a multivariable control method with wave-prediction and passivity technique is proposed for real-time systems in the presence of internet delay dynamics. A synchronization scheme for nonlinear dynamical networked systems including delays and uncertainty is designed using passivity analysis [13]. A two-layer control scheme has been discussed for maintaining the stability of BTSs [56]. This scheme consists of one layer to assure the transparency and a second one for handling the passivity of the system. In 2010, K. Hertkorn et al. [57], a general time-domain passivity control approach is applied for a haptic system with multi-degree of freedom. Other examples on this approach including stabilizing the energy or position drift compensation can be found in [58]-[63].

Some advantages of this approach in the literature include: applying switching method for energy dissipation [64] or independent of time-varying delay [65], the combination of passivity and transparency [56], applying it to non-ideal system [61], applying both power-based and energy-based approaches [60], and the elimination of system uncertainties [66].

On the other hand, some drawbacks of this approach include: weak transparency [64], the transparency is not guaranteed in the passivity layer [56], limited application and no consideration of delay [61], applied to a system with constant delay [58], and limited random delays [60]. More examples on the passivity-based control approach are [2], [14], [15], [67]-[71].

\subsection{Wave variable-based control}

The first introduction of the wave variable-based control approach was in 1991 by Niemeyer and Slotine [72].

The following transformation is applied to the reference signals in the BTS, i.e. joints velocity and external forces $\dot{q}_{i}$ and $f_{j}, i \in\{m, s\}$ and $j \in\{e, h\}$ to obtain a wave formulation shown in (12) and (13).

$$
\begin{aligned}
& u_{m}=\frac{1}{\sqrt{2 b}}\left(f_{h}^{*}(t)+b \dot{q}_{m}(t)\right) \\
& u_{s}=\frac{1}{\sqrt{2 b}}\left(f_{e}(t)-b \dot{q}_{s}^{*}(t)\right)
\end{aligned}
$$

Now, the new signals $u_{m}$ and $u_{s}$ are sent by the communication channel. Additionally, the desired signals for tracking will be $f_{h}^{*}$ and $\dot{q}_{s}^{*}$, and the communication channel has a characteristic impedance $b$. So, the flow of power in the communication channel between the master and slave robots are given by (14):

$$
\begin{aligned}
P_{\text {in }}(t) & =f_{h}^{* T}(t) \dot{q}_{m}(t)-f_{e}^{T}(t) \dot{q}_{s}^{*}(t) \\
& =\frac{1}{2}\left(u_{m}^{T}(t) u_{m}(t)-v_{m}^{T}(t) v_{m}(t)\right)+\frac{1}{2}\left(u_{s}^{T}(t) u_{s}(t)-v_{s}^{T}(t) v_{s}(t)\right)
\end{aligned}
$$

where $v_{m}(t), v_{s}(t)$ are the received signals and $u_{m}(t), u_{s}(t)$ are the reference signals on the master and slave sides of a BTS satisfying by (15) and (16):

$$
\begin{aligned}
f_{h}^{*}(t) & =b \dot{q}_{m}(t)+\sqrt{2 b} v_{m}(t) \\
\dot{q}_{s}^{*}(t) & =\frac{1}{2}\left(\sqrt{2 b} v_{s}(t)-f_{e}(t)\right) .
\end{aligned}
$$


The researchers have developed and designed controllers for BTSs in the wave domain due to the fact that intrinsic passivity is preserved in the wave formulation [73]-[79]. However, the passivity could be unsaved in the power signals. According to this feature, it is important to build and place a passive filter at the master robot for estimating the model of the slave robot and enhance the force reflection in haptic applications. A similar proposal was provided for constant known delays [78], [79].

An incident named wave reflection occurs when the characteristic impedance of the network channel is not similar to that of the ports of the master and slave. Since it has a high impact on the BTS performance, wave reflection must be handled cautiously in the system design. Normally, the impedance matching element $b$ is used for tuning this feature at both sides of the communication channel. But, the performance of the BTS, mainly the position tracking could be affected by this parameter. To overcome this scenario and decrease position drift, parameter $b$ is not considered on the master side [79]. To notice the difference between these two ways, the position tracking drifts for the master side when $b$ is removed is given by (17) and (18):

$$
q_{m}^{*}\left(t-d_{1}(t)\right)-q_{s}(t)=\frac{1}{2 b} \int_{t-2 d_{1}(t)}^{t} f_{e}^{*}(\eta) d \eta
$$

and when $\mathrm{t}$ is not removed

$$
q_{m}^{*}\left(t-d_{1}(t)\right)-q_{s}(t)=\frac{1}{b} \int_{0}^{t} f_{e}^{*}(\eta) d \eta+q_{s}(t) .
$$

It is noted here that as $b$ increases, the position drift decreases. Nevertheless, since more damping is introduced, the BTS will have a poor performance.

Some advantages of this approach in the literature include: the improvement of position tracking [73], optimization of wave transformation [77], the improvement of tracking and power optimization [80], dealing with passive and non passive human model [76], and guaranteed passivity [75]. The drawbacks of this approach include: the existence of estimation error [73], no consideration of time delay [77], [80], reducing the transparency [76], and considering constant time delay only [75].

\subsection{Adaptive control approach}

The structure of BTSs includes several important parameters and model uncertainties to be concerned by designers. These parameters are revealed in all of the elements of the BTS shown in Figure 1, i.e. the master and slave robots, the communication network, the human operator, and the environment.

The adaptive control approach is widely applied by researchers to solve the problem of the existence of the uncertainties in BTSs. Moreover, a lot of works and efforts were invested in this approach due to the linearity of parameters of BTSs as noted in (1).

To understand this method, the general design of the adaptive controllers for the BTSs is given by (19) and (21). The human operator and the environment are presented as a general nonpassive nonhomogeneous force as follows (19):

$$
\begin{gathered}
\hat{f}_{j}(t)=\hat{M}_{j}(t) \ddot{x}_{j}(t)+\hat{D}_{j}(t) \dot{x}_{j}(t)+\hat{K}_{j}(t) x_{j}(t)+\hat{f}_{j 0}(t) \\
\hat{M}_{i}\left(q_{i}\right) \ddot{q}_{i}+\hat{C}_{i}\left(q_{i}, \dot{q}_{i}\right) \dot{q}_{i}+\hat{g}_{i}\left(q_{i}\right)+\delta(t)=\tau_{i}+f_{j}
\end{gathered}
$$

where $\hat{M}_{j}, \hat{D}_{j}, \hat{K}_{j}$, and $\hat{f}_{j 0}$ are the estimation parameters of the mass, damping, stiffness, and nonhomogeneous parameters of the human operator, $i \in\{m, s\}$ represents master and slave systems, and $j \in\{h, e\}$ represents the human and environment, respectively.

Remark 2. The same philosophy could be applied for external disturbance rejection inserting a bounded term representing the external disturbance $\delta(t)$, as $(20)$ :

$$
\|\delta(t)\| \leq \Delta<\infty
$$

In this scenario, an adaptive parameter $\hat{\delta}(t)$ is needed to be added to the control signal in the BTS (1) such that (21) and (22):

$$
\tau=\hat{f}_{j}(t)+\hat{M}(t) a+\hat{C}(q, \dot{q}) \dot{q}+\hat{g}(q)+\hat{\delta}(t)
$$


in addition to the term of the PD controller

$$
a=\ddot{q}_{d e s}-K_{d} \dot{e}-K_{p} e
$$

where $K_{d}, K_{p}>0$ are symmetric matrices.

Then, after some manipulation and linear parameterizing, the dynamics of the BTS are formulated as

$$
\tilde{M}\left(\ddot{e}+K_{d} \dot{e}+K_{p} e\right)=\Psi(q, \dot{q}, \ddot{q}) \tilde{\theta}(t)+\tilde{\delta}(t)+\tilde{f}_{j}(t)
$$

with the estimation error of the corresponding parameter is indicated by the sign $\sim$. So, these estimated parameters have to be handled in the design of the controller to achieve or maintain the stability and the task performance of the BTS in the presence of uncertainties. The adaptive law for $\tilde{\theta}(t)$ and $\tilde{\delta}(t)$ is obtained by applying the stability analysis such as the Lyapunov theorem such that the convergence of the error signals to zero is achieved and the estimation errors are bounded [34].

Additionally, one of the general methods in adaptive control design is to utilize the linearity in the parameters of the BTS (1). Let the combined error signals of both position and velocity of the master and slave be defined as (24) and (25):

$$
\epsilon_{m}=\dot{e}_{m}+P_{m} e_{m}
$$

and

$$
\epsilon_{s}=\dot{e}_{s}+P_{s} e_{s}
$$

where $P_{m}, P_{s}>0$, so, the control signals are (26):

$$
\begin{aligned}
u_{i} & =-u_{i d}-\hat{M}_{i}\left(q_{i}\right) P_{i} \dot{q}_{i}-\hat{C}_{i}\left(q_{i}, \dot{q}_{i}\right) P_{i} q_{i} \\
& =-u_{i d}-\Psi_{i} \hat{\theta}_{i}, \quad i \in\{m, s\}
\end{aligned}
$$

and the closed loop BTS will be (27):

$$
M_{i} \dot{e}_{i}+C_{i} e_{i}=\Psi_{i} \tilde{\theta}_{i}+u_{i d}-f_{j}, \quad i \in\{m, s\}, j \in\{h, e\}
$$

will guarantee both of the errors' asymptotic convergence and the parameter estimation errors $\tilde{\theta}_{i}$ to be bounded. Also, the Lyapunov analysis or any other stability analysis could be used to derive the adaptation laws $\dot{\hat{\theta}}_{i}$.

Remark 3. The adaptive control approach could be combined with intelligent and some nonlinear algorithms like fuzzy control and neural network (NN). This feature allows researchers to design new algorithms to enhance and improve the performance of the BTSs which makes it one of the most powerful control approaches.

The designed control system using the combination of algorithms leads to a more robust and efficient BTS. Moreover, it is not necessary to have the exact model and precise information about the BTS. Also, these algorithms allow overcoming other restrictions in the BTSs, for example, long time-varying delays, input saturation, packet loss, force reflection error, and position drift.

An adaptive switched control considering passive and non-passive external forces, actuator saturation, and unknown dynamics is designed for BTSs including asymmetric time-varying delay [81]. The obtained scheme has the capability of adaptive systems which is indicated by the achieved bounded position tracking error of the BTS. An adaptive fuzzy control system was developed to attain the state-independent input-tooutput stability for a multilateral asymmetric teleoperation system [82]. Also, similar works are found such as [83]-[85].

Chen et al. [86], an adaptive finite-time control method is presented using subsystem decomposition. The stability is guaranteed by applying the Lyapunov-Krasovskii analysis and bounded tracking error is obtained. Robust adaptive techniques were proposed for considering unknown parameters the uncertainties of the BTS [87]. More examples on this approach are [8]-[13], [41].

Some advantages of the adaptive control approach in the literature include: considering non-homogenous and state independent input/output stability [81], [82] non-passive input forces [24], considering drift diffusion improved haptic [88], and multi robot slave and input saturation [89]. The drawbacks of this approach include: chattering of torque [81], [82], [89], does not consider time delay [88], constant time delay [90], and non assymptotic stability [24], [87]. 


\subsection{Robust control approach}

The main objective when applying the robust control approach is to consider the worst conditions which have negative effects on the stability and the performance of the BTSs. So, the control scheme is called robust when it maintains the stability and the performance of the BTSs affected by disturbing factors. In addition to the elements affecting normal systems such as the uncertainties in the model, neglected dynamics, and external disturbances, the network-induced uncertainty is the most tragic element that affects the stability and performance in BTSs.

In mathmatic words, the robust control problem is minmax problem such that it is a minimization of the fraction $y_{\delta}=\|y\| /\|\delta\|$ while the term $y_{x}=\|y\| /\|x\|$ is maximized, with $y_{\delta}$ refers to the disturbances' contribution $\delta$ in the output of the system $y$, with the desired input $x .\|$.$\| is any Euclidean norm function. Ideal$ value for $y_{\delta}$ is 0 and for $y_{x}$ is 1 .

The sliding mode control (SMC) is the most familiar robust control approach. In this technique, the controller is designed to achieve the convergence of the error signal to a predetermined sliding surface. This surface is a differential equation for the error which has a solution lying on a convergent set or point. One way for SMC is to define the error between the motion of the master and slave robots as $e=x_{s}-K x_{m}$. So, the sliding surface is given by (28):

$$
s=\dot{e}+\lambda e
$$

in which $\lambda>0$ is a constant used for determining the features of the surface and the error's rate of convergence, and $K>0$ is a coefficient matrix for scaling.

Remark 4. A dissipation condition must be met to guarantee the robust stability as the following foe example:

$$
s \dot{s} \leq-\gamma\|s\|<0
$$

where $\gamma>0$ is a constant. Either large gain or chattering control signals will be generated to obtain this feature.

A chattering-free SMC approach is presented to achieve a robust performance of a BTS in uncertain conditions [91]. The number of the needed sensors is reduced by applying a pseudo-sensorless approach which also decreased the uncertainties affecting the BTS. A PD controller is proposed for specific delayed BTSs using the linear matrix inequalities (LMI) technique [92]. The BTS has been stabilized regardless of the occurrences of delays in the communication channel. But, the presented PD controller was applied only on the slave robot and designed for the constant time delay.

The robust control approach is more suitable for linear systems because of the norm-based analysis and calculations. So, Some techniques of the robust control like $H_{2}, H_{\infty}$, and $\mu$-synthesis are applied for linearized time delay BTSs. These techniques are normally used in the frequency domain to handle the worst case scenario, uncertainties, and the upper limit of the delay of the linearized BTSs.

Some advantages of the adaptive control approach in the literature include: reducing time sensitivity and convex optimization using $H_{\infty}$ approach [93], achieving optimal transparency using $H_{2}-H_{\infty}$ approach [94], maintain passivity [86], considering time varying uncertainties [95], and simultaneous stability and transparency [96]. The drawbacks of this approach include: considering linear model with constant delay [93], [94], [97], linear model without time delay [95], occurrences of chattering torque [86], [96], constant time delay [98]-[100].

\subsection{Neural network-based approach}

One of the worst features affecting the stability and the performance of BTSs is the existence of timevarying delays. Some literature considering this situation by considering the input saturation with time-varying delay using adaptive fuzzy control method [101] or applying two controllers, a PD-like and P-like controllers to handle constant delay and time-varying delay, respectively [102].

The neural network (NN) based approach for controlling BTSs has a great value due to its high potential to learn and parallel adaptive processing. These features make the NN more practical for complex nonlinear BTSs and suitable to deal with the time delays and uncertainties existing in the BTSs. The basic idea of NNs comes from the neuron system in the human body. So, similar to the cell model, they have dendrite weights $w_{j}$, a nonlinear function $\sigma($.$) which is normally called the activation function of the cell as shown in (29), and$ 
a firing threshold $w_{0}$. Let the output signal of the $n$-dimensional input signal $x(t) \in \mathbb{R}^{n}$ to be $y(t)$. So, the neuron is represented by the following (29):

$$
y(t)=\sigma\left(\sum_{j=1}^{n} w_{j} x_{j}(t)+w_{0}\right) .
$$

The insight of the neuron is excitatory synapses when $w_{j}>0$ and it is inhibitory synapses when $w_{j}<0$ [103]. The activation function $\sigma($.$) is introduced separately and related to the requirements of the$ current application. It also shows the behavior of the cell in the case that $y(t)$ is in its range. Also, since the derivative is required in learning algorithms for many cases, $\sigma($.$) has to be differentiable. Both the input vector$ and weights vector are assigned to derive the output signal. So, we will get the following (30):

$$
\begin{aligned}
y(t) & =\sigma\left[w_{0}, w_{1}, \ldots, w_{n}\right]\left[1, x_{1}, \ldots, x_{n}\right]^{T} \\
& =\sigma\left(\overrightarrow{\mathbf{w}}^{T} \overrightarrow{\mathbf{x}}(t)+w_{0}\right) .
\end{aligned}
$$

Remark 5. For the case of the multicell model of a neuron consists of $r$ cells, with an input signal $\overrightarrow{\mathbf{x}}(t)$ and an output $y_{m}(t)$, the system is represented by the following (31):

$$
\overrightarrow{\mathbf{y}}(t)=\left[y_{1}, y_{2}, \ldots, y_{m}\right]^{T}=\vec{\sigma}\left(W^{T} \overrightarrow{\mathbf{x}}(t)+\overrightarrow{\mathbf{w}}_{0}\right)
$$

in which the weight matrix is given by (32):

$$
W=\left[w_{i j}\right], i=1 \ldots r, j=1 \ldots n .
$$

The features of the NNs allow researchers to apply them in a wide range of applications to solve the nonlinear and uncertainty problem for both control and estimation problems especially in robotics and BTSs [66], [89], [104], [105]. A comprehensive discussion on the application of NNs for robotics control is presented in [103].

NNs were used in BTSs for approximating and dealing with delays and uncertainties of the communication channel. So, NNs are applied for modeling the delay in the system $d_{f}(t)$ and $d_{b}(t)$, or the total transmission between the master and the slave. Let us assume that the position signal of the master robot $x_{m}(k)$ has to be transmitted to the slave side at the sampling time $k$. One can develop the NNs for modeling the network to estimate this process as follows (33):

$$
\hat{x}_{m}(k)=T_{\mathrm{NN}}\left(x_{m}(i)\right), i \in[1,2, \ldots, k-1, k]
$$

with $T_{\mathrm{NN}}($.$) represents the NN model obtained by (34):$

$$
T_{\mathrm{NN}}\left(x_{m}(i)\right)=\vec{\sigma}\left(W^{T} x_{m}(i)+\overrightarrow{\mathbf{w}}_{0}\right) .
$$

The local controllers on the master and slave sides are designed to provide a prediction on the transmitted signals using the estimator model of the communication channel. So, the control signal in this system will be more efficient in comparison to the normal case where the signals received by the controllers could be affecting by the delay or the distortion in the system [34].

A prediction algorithm using adaptive linear NN was developed for the application of an internet time delay system [106]. Auld et al. [107], a Bayesian NN was implemented for classifying the internet traffic without information about the IP. The proposed method provided $95 \%$ accuracy after testing it on a real training $\mathrm{NN}$ for 8 months. A recurrent $\mathrm{NN}$ was utilized for modeling and predicting the Internet end-to-end delay [108]. The recurrent NN was trained and validated using the discrete-time data obtained by measuring the delay between two different nodes. NN approach was also implemented for modeling traffic flow in selfsimilar computer networks which is statically do not depend on time [109]-[112]. Other examples including further details could be found in [113]-[115].

In addition to the aforementioned applications, NNs were implemented in the local controllers on both sides of the BTSs. A better performance was obtained by combining the NN approach with other control methods, mainly the adaptive control method [66], [89], [104]. Another application of the NN in the BTSs is to 
utilize the $\mathrm{NN}$ in designing the control system for estimating the delayed or disturbed signals and for protecting the system simultaneously when it is subjected to uncertainties and latency [34]. However, it is worth knowing that the parameters of the NN structure such as the number of layers, the number of neurons, etc. affect the accuracy of the estimation process. So, the application of the NN control approach handle the uncertainties and delay indirectly by giving the best possible result within the unknown circumstances of the system. A radial basis function (RBF) NN-based procedure was proposed for handling variable delays in teleoperation system [36]. The model contains linear viscous and Coulomb velocity-dependent frictions. But, it is required to measure the acceleration or to estimate it, and this is not practical in many cases. The following RBF Gaussian functions were applied for approximating the dynamics of the BTS (1):

$$
\Phi_{n}(x)=\exp \left(-\frac{1}{2 H_{n}^{2}}\left\|x-C_{n}\right\|^{2}\right)
$$

where $H_{n}$ is the width and $C_{n}$ is the center for the $n$th neuron.

$$
\operatorname{Dyn}_{i}\left(\mathbf{x}_{i}\right)=W_{i}^{T} \Phi_{i}\left(\mathbf{x}_{i}\right)+\xi_{i}\left(\mathbf{x}_{i}\right)
$$

where $\mathbf{x}_{i}=\left[\ddot{q}_{i}^{T}, \dot{q}_{i}^{T}, q_{i}^{T}, \ddot{x}_{i}^{T}, \dot{x}_{i}^{T}, x_{i}^{T}\right]^{T}$. Therefore, the following approximated dynamic are implemented in the control system to overcome both of the unknown bounded terms and the inverse dynamics by (37):

$$
\operatorname{Dyn}_{i}\left(\mathbf{x}_{i}\right)=f b_{i}\left(q_{i}, \dot{q}_{i}\right)+g_{i}\left(q_{i}\right)+C_{i}\left(q_{i}, \dot{q}_{i}\right) \dot{q}_{i}+M_{j} \ddot{x}_{i}+B_{j} \dot{x}_{i}+K_{j} x_{i}+D_{i}(t) .
$$

Thus, the control signal $\tau_{i}$ is calculated for the following system shown in (38):

$$
\begin{aligned}
& M_{i}\left(q_{i}\right) \ddot{q}_{i}+C_{i}\left(q_{i}, \dot{q}_{i}\right) \dot{q}_{i}+g_{i}\left(q_{i}\right)+f b_{i}\left(q_{i}, \dot{q}_{i}\right)+D_{i}(t) \\
& \quad=\tau_{i}+M_{j} \ddot{x}_{i}+B_{j} \dot{x}_{i}+K_{j} x_{i}+f_{j 0}+\operatorname{Dyn}_{i}\left(\mathbf{x}_{i}\right) \\
& \quad=\Psi_{i}\left(\mathbf{x}_{i}\right) \Theta_{i} .
\end{aligned}
$$
using (39):

The desired control signal for system (38) when the function $\operatorname{Dyn}_{i}$ is ideally approximated, is obtained

$$
\tau_{i}=M_{i}\left(q_{i}\right)\left(\ddot{q}_{i}^{*}-K_{D i}\left(\dot{q}_{i}-\dot{q}_{i}^{*}\right)-K_{P i}\left(q_{i}-q_{i}^{*}\right)\right)
$$

with positive-definite gain matrices $K_{D i}$ and $K_{P i}$ that by substituting in (38) leads to a Hurwitz function show in (40):

$$
\ddot{e}_{i}+K_{D i} \dot{e}_{i}+K_{P i} e_{i}=0 .
$$

The NN-based approximations are inherently intelligent enough to use the previous experiences of the BTS for learning, although they have an error in real applications. The accuracy of the approximation could be increased by selecting large quantities for $\mathrm{NN}$ nodes $n$ in (35) for function $\operatorname{Dyn}(x)$ over a compact set of the input $x \in \Omega_{x} \subset \mathbb{R}_{x}$. Other methods of control were combined with $\mathrm{NN}$ to decrease the unavoidable estimation error. A neural adaptive control was proposed for a single-master multi-slave BTS [89]. The use of the NNvto model of the unknown nonlinear plants helped to handle the uncertainties in the dynamics of and the input of the BTS. But, several assumptions were applied in this scheme such as the singularity-free motion of the slave robot, the rigidity of the object, and rigid attachment between slaves' end-effector and the object.

Consider the signals of the combined error to be (41):

$$
\begin{aligned}
r_{m} & =\dot{x}_{m}+\Gamma_{m} e_{m} w, \dot{x}_{m r}=-\Gamma_{m} e_{m} \\
r_{s} & =\dot{x}_{s}+\Gamma_{s} e_{s}, \dot{x}_{s r}=-\Gamma_{s} e_{s}
\end{aligned}
$$

with error definitions $e_{m}(t)=x_{m}(t)-x_{s}\left(t-d_{b}(t)\right)$ and $e_{s}(t)=x_{s}(t)-x_{m}\left(t-d_{f}(t)\right)$, and matrices $\Gamma_{m}, \Gamma_{s}>0$. Then, the adaptive control signal is selected as (42):

$$
\begin{gathered}
u_{m}=-\beta_{m} \hat{\Theta}_{m}^{T} \Phi_{m}\left(Z_{m}\right) \operatorname{sgn}\left(r_{m}\right) \\
u_{s}=-\beta_{s} \hat{\Theta}_{s}^{T} \Phi_{s}\left(Z_{s}\right) \operatorname{sgn}\left(r_{s}\right)
\end{gathered}
$$


where $Z_{m}$ and $Z_{s}$ are the state-based vectors with elements, such that $\left\|\dot{x}_{m}\right\|$ and $\left\|\ddot{x}_{m r}\right\|$, and $\left\|\dot{x}_{s}\right\|$ and $\left\|\ddot{x}_{s r}\right\|$, respectively. The parameter estimated vectors $\hat{\Theta}_{m}^{T}$ and $\hat{\Theta}_{s}^{T}$ with the adaptation signal is represented as (43):

$$
\begin{aligned}
& \dot{\hat{\Theta}}_{m}=-a_{m} \hat{\Theta}_{m}+\omega_{m}\left\|r_{m}\right\| \Psi_{m} \\
& \dot{\hat{\Theta}}_{s}=-a_{s} \hat{\Theta}_{s}+\omega_{s}\left\|r_{s}\right\| \Psi_{s}
\end{aligned}
$$

where $a_{m}, a_{s}, \omega_{m}$, and $\omega_{s}$ are positive controller designed parameters. The adaptive system (43) is used to update the parameters of model (38), So the error in the system (41) will converge to zero by applying the $\mathrm{NN}$-adaptive control structure (42).

Another challenge in BTSs is the problem of inverse kinematics of serial robots' manipulators. An NN-based control scheme designed while considering kinematic uncertainties for a multi-master single slave BTS [105]. The error was minimized by estimating the Jacobian matrix that relates the joints' and Cartesian velocity of the slave robot by considering the constraints affecting the workspace. Some advantages of this approach in the literature include: applying NN for modeling backlash-like hysteresis and obtaining smooth torque [104], considering kinematic uncertainties with Markovian time delay [105], considering unknown varying delay [66], and handling input saturation and achieving smooth estimation [89]. The drawbacks of this approach include: the obtained tracking error is non-asymptotic [104], the delay is state dependent [105], did not consider the existence of external disturbances [66], and exist of chattering in the obtained torque [89].

\subsection{Fuzzy control approach}

The fuzzy control was proposed by Zadeh [116]. The idea of the fuzzy control approach is applying fuzzy sets for developing a decision algorithm. It mainly includes several fuzzy if-then rules which are applied to find the system's output related to each set of input. There are four elements for each fuzzy system: inference rules, fuzzifier, defuzzifier, and membership functions. Let us consider the following example of a statement that describes the $k$ th rule $R^{(k)}$ in which the membership function is Gaussian, the fuzzifier is a singleton, and the defuzzifier is center average

$R^{(k)}: \operatorname{IF} x_{1}$ is $\mathbb{A}_{1}^{K}$ and $\ldots$ and $x_{n}$ is $\mathbb{A}_{n}^{k}$, then $y$ is $\mathfrak{B}^{k}, k=1,2, \ldots, K$ with the linguistic variables associated with the system inputs defined by $\overrightarrow{\mathbf{x}}=\left[x_{1}, x_{2}, \ldots, x_{n}\right]^{T} \in U \subseteq \mathbb{R}^{n}$ and output of the fuzzy logic system $y \in \mathbb{R}$. Also, the inputs from the set $\mathbb{A}$ to the outputs in the set $\mathbb{B}$ is mapped by the fuzzy system using a nonlinear algorithm.

The fuzzy control approach is an efficient method for the approximation and modeling of complex and non-linear BTSs. A two-layer fuzzy controller was designed to protect a remote mobile robot in the case of a failure in the communication [117]. A multilevel fuzzy approach is implemented to enhance the robustness of the fuzzy system bu using several hierarchical fuzzy rules [118]. In 2016, Zhai and Xia [83] an adaptive fuzzy control system using a switching strategy is presented for the application of multi-robot cooperation affected by time-varying delays. In the same direction, an adaptive fuzzy controller is proposed for synchronizing multirobot BTSs including random delays and dynamic uncertainties [84]. The robustness and the stability of the BTS were achieved by applying the LMI-based Lyapunov-Krasovskii method under stochastic circumstances. The membership functions were selected to be Gaussian such that $\mu_{F i}^{j}=\exp \left(-\frac{\left(x_{j i}-c_{j i}\right)^{2}}{\sigma_{j i}^{2}}\right)$. A fuzzy control system was designed for a BTS including friction forces [85]. The defuzzifier is chosen as center average and the fuzzy outputs are given by the following (44):

$$
y(\overrightarrow{\mathbf{x}})=\frac{\sum_{j=1}^{l} y^{i}\left(\prod_{i=1}^{n} \mu_{F i}^{j}\left(x_{i}(t)\right)\right)}{\sum_{j=1}^{l} \prod_{i=1}^{n} \mu_{F i}^{j}\left(x_{i}(t)\right)}
$$

with linguistic variables $x_{i}(t)$ and maximum value of $y^{j}$ for each $\mu_{F i}^{j}$, so, $\mu_{F i}^{j}\left(y^{j}\right)=1$.

Using the fuzzy basic functions

$$
y(\overrightarrow{\mathbf{x}})=\theta^{T}(t) \nu(\overrightarrow{\mathbf{x}})
$$

in which

$$
\theta(t)=\left[y^{1}(t) y^{2}(t) \ldots y^{l}(t)\right]^{T}
$$




$$
\nu(\overrightarrow{\mathbf{x}})=\left[\nu_{1}(\overrightarrow{\mathbf{x}}) \nu_{2}(\overrightarrow{\mathbf{x}}) \ldots \nu_{l}(\overrightarrow{\mathbf{x}})\right]^{T}
$$

and

$$
\nu_{j}(\overrightarrow{\mathbf{x}})=\frac{\prod_{i=1}^{n} \mu_{F i}^{j}\left(x_{i}(t)\right)}{\sum_{j=1}^{l} \prod_{i=1}^{n} \mu_{F i}^{j}\left(x_{i}(t)\right)} .
$$

(45):

Knowing the universal approximation theorem, the estimation of the dynamic equation $\operatorname{Dyn}(\overrightarrow{\mathbf{x}})$ is

$$
\theta^{*}=\arg \min _{\theta \in \Omega_{\theta}}\left(\sup _{\overrightarrow{\mathbf{x}} \in \Omega_{\overrightarrow{\mathbf{x}}}}\left|\theta^{T}(t) \nu(\overrightarrow{\mathbf{x}})-\operatorname{Dyn}(\overrightarrow{\mathbf{x}})\right|\right)
$$

with proper sets $\Omega_{z}$ for $z(t)$ and $\Omega_{\theta}$ for $\theta(t)$. Here, the minimum estimation error $\|\epsilon(\overrightarrow{\mathbf{x}})\| \leq \epsilon^{*}, \epsilon^{*}>0$ is similar to that obatained in NN-based approach shown in (46):

$$
\operatorname{Dyn}(\overrightarrow{\mathbf{x}})=\theta^{*} \nu(\overrightarrow{\mathbf{x}})+\epsilon(\overrightarrow{\mathbf{x}})
$$

Also, the parameter $\theta(t)$ is updated by applying the adaptive algorithms such that it reach $\theta^{*}$ and reducing $\epsilon(\overrightarrow{\mathbf{x}})$.

Some advantages of the fuzzy control approach in the literature include: reducing of the chattering and maintain the transparency [119], considering Markovian time-delay [84], [120], convergance of the state [121], state independent input/output stability [83], and asymptotic tracking [85]. The drawbacks of this approach include: considering constant time delay [85], [119], simulated delay [120], linear model and constant time delay [121], chattering torques [83], and decoupled master-slaves [84].

\section{CYBER ATTACKS IN BTSS}

In this section, we will discuss the control problem of BTSs in the presence of DoS and deception attacks. First, we will explain the effects of DoS and deception attacks on the BTS. Then, we will propose the stability control problem of such a BTS in several circumstances.

\subsection{Background}

The communication among the items of BTSs, mainly the master's and slave's controllers, are occurred through a common network medium as shown in Figure 3. It is important to secure this network to prevent possible dangerous attacks during the communication of data. As explained above, the BTS could face instability or it is led to undesired operations by these attacks. Thence, it is essential to design controllers for BTSs while taking into accounts the security issues [122], [123].

Cyber attacks have two main types from a control engineering design viewpoint: 1) Denial of service (DoS) attack, which is a strategy that is often applied to occupy the communication resources to prevent the communication of signals among the system elements. 2) Deception attack, which is the modification of the data integrity for the communicated signals in the BTS [124].

Figure 3 shows a framework of a common teleoperation system including: an operator, master robot, master controller, communication network, slave controller, and slave robot. The position and velocity signals are sent between the master and slave controller through a communication channel. The network could be in any suitable wired or wireless structure such as a wide area network (WAN), the Internet, cellular, Ethernet, Wi-Fi, or a local area network (LAN). This network is affected by several cyber attacks in the forward path (the signal transmitted from master controller to slave controller) and/or the backword path (the signal transmitted from slave controller to master controller).

In the presented framework, a teleoperation controller is designed such that the end-effector of the slave robot follows the corresponding position of the master robot. The interaction of the sub-task controller and the teleoperation controller is designed using theoretical formulations to achieve the stability for the closedloop BTS [36]. Also, the human operator will know the remote environment through the feedback signals sent by the slave side. So, the human operator's work is to control the slave manipulator and the slave robot has the capability to track the master robot and perform an extra sub-task autonomously. Due to the aforementioned importance of controlling systems in the presence of cyber attacks, many literature consider this urgent and major problems. Many literature discussed the DoS attacks alone [125]-[130], deception attacks alone [131], [136], or two attacks [123], [124], [137]-[139]. 


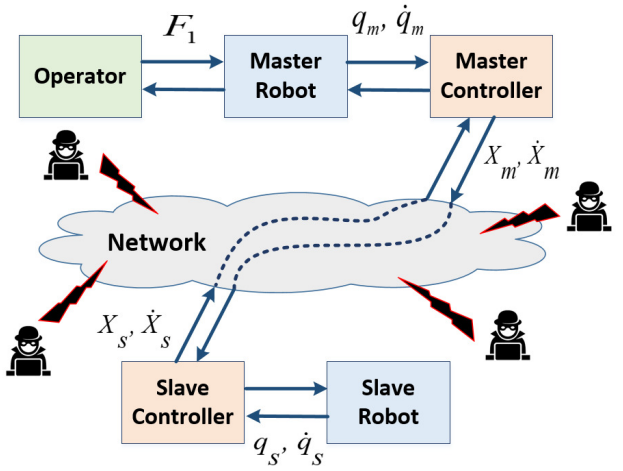

Figure 3. Framework of a teleoperation system with cyber attacks

\subsection{Literature on cyber attacks in BTSs}

In section 4.2, we will present and discuss the literature that considers cyber attacks in the control of BTSs. One of the initial work that was to discuss the security of Telesurgical Robot Systems (TRSs) in the presence of unreliable network [140]. TRSs include critical operations in difficult situations or environments like disaster territories, underwater, and battlefields. In these situations, it is necessary to apply wireless communication. Thus, some challenges are introduced in TRSs including: delay in the signal, maintain a predefined threshold for the reliability, and providing several security measures that depend on the environment and the nature of the operation.

An adaptive information coding method was designed to support both confidentiality and adaptive reliability simultaneously [140]. In this method, both light-weight privacy and adaptive reliability are integrated by using information coding in a single protocol named Secure and Statistically Reliable UDP (SSR-UDP). Another issue in the TRSs is to enhance the security of Interoperable Telesurgery Protocol (ITP) which is achieved by the collaboration between researchers in TRSs and security. The structure for the communication between the telesurgery robots and their controllers is determined by the ITP. A proof of concept and framework of Secure ITP was designed and presented [141]. In this project, open-source software tools were implemented for building a prototype for security enhancement. This prototype could be used to develop and demonstrate new security technologies in the field of TRSs.

The possible class of attacks which may affect rescue robotic systems were explained [42]. These types of attacks may occur as a result of the Internet-enabled reach-back process. The set of required security and performance needed by rescue robots was discussed. Moreover, a method for mitigating this kind of attack was proposed. Many security threats may affect surgical teleoperation systems (STSs) since they use wireless communication, antagonistic operating environments, and work in a critical time operation. [43] analyzes some of the threats and discusses their impact. The threats affecting mobile STSs are classified as [43]: label

(a) Attacks affecting the wireless communication: an unencrytpted wireless channel renders mobile surgical telerobotic systems could be affected by several attacks such as:

- Eavesdropping.

- Jamming.

- Message modification/False data injection.

(b) Attacks targeting surgeon-manipulator interaction: attacks that affect the wireless medium, including eavesdropping and message modification, are mainly mitigated by applying encryption algorithms. The STSs are rendered to a man-in-the-middle (MTIM) attack due to the lack of an authentication mechanism among them, which can be as follows:

- Simple replay.

- Delay attack.

- Message dropping.

- Combined delay and drop attack.

- Message modification and spoofing attack.

(c) Attacks on the surgeon-side software (such as attacks exploiting development (engineering) interfaces). 
(d) Attacks on the manipulator-side software (such as attacks on the programmable logical controllers (PLC)).

(e) Physical attacks.

In 2015, Bonaci, Yan, Herron, Kohno, and Chizeck [144] analyzes cyber-security attacks against one of the advanced TRSs, Raven II R. Possible attacks were classified, and more attention was given to the denialof-service (DoS) attacks, which cannot be avoided by implementing the existing cryptographic methods. The impact of these attacks on the TRSs was discussed using a set of experiments including human subjects. Also, possible steps to relieve the recognized DoS attacks were considered, and the suitability of these methods for TRSs was evaluated. A model-based analysis framework for estimating the consequences of control signals by computing the dynamic of the BTS in real-time is discussed [145]. The directed cyber attacks on TSRs which aim to affect the system by inserting malicious control signals during surgery were demonstrated. It is known that these kinds of cyber attacks could affect the physical system such as causing a sudden motion of the robot's arm leading to affect the patient or damaging the robot or some of its parts during the surgery itself. Figure 4 shows a typical structure for controlling surgical robots. In Figure 5, the kinematics chain in the tested robot RAVEN II control software is presented including all possible attacks.

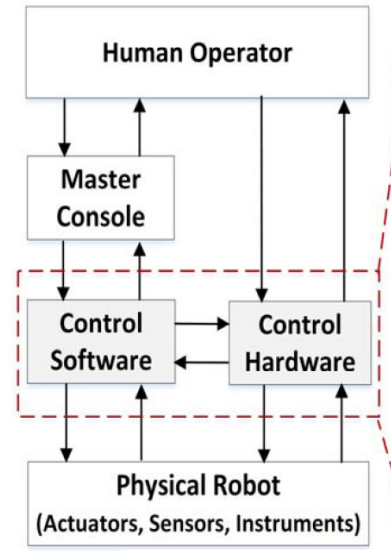

(a)

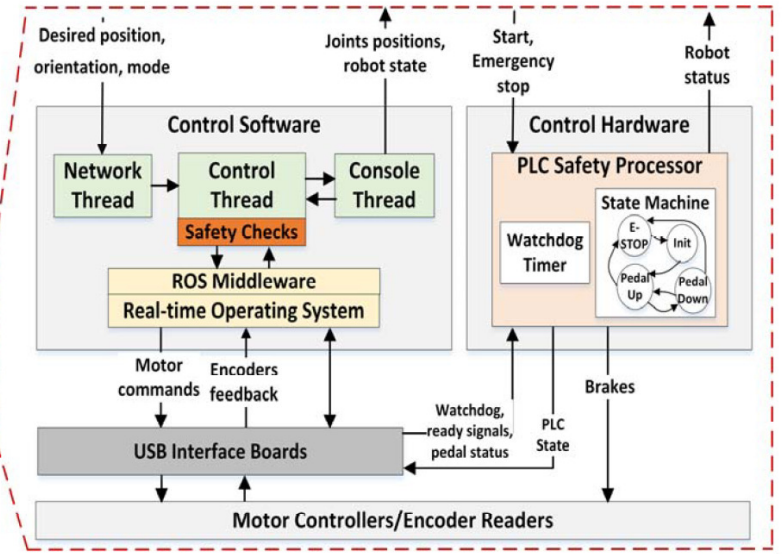

(b)

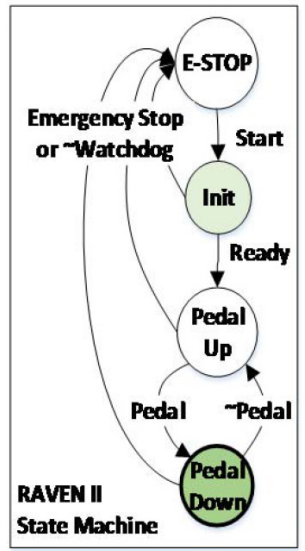

(c)

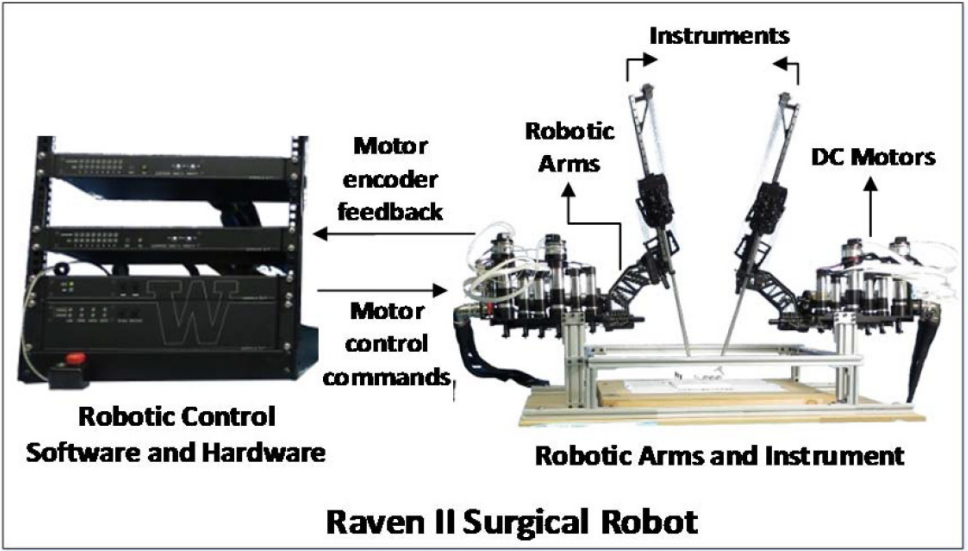

(d)

Figure 4. Typical structure for controlling surgical robots: (a) The control structure of a TSR, (b) Control loops in the software and hardware in the RAVEN II, (c) operational state machine of the RAVEN II,

(d) The surgical platform of RAVEN II. [145]

The basic architecture of an industrial robot is examined and a coherent deployment from a systems security point of view was analyzed [146]. Also, a model for the attacker is presented and compared with the minimum requirements' set for industrial robots such as the precision to sense the surrounding environment, the correction in control logic execution, and the safety of human operators. 
Using a practical experiment, it was shown how the proposed designed attacker can achieve the requirements by exploiting the software vulnerabilities. Also, the standard for safety and other challenges of security in industrial robotics were discussed [146].

A task-space control structure for BTSs with uncertainty in kinematic/dynamic, time-delay, and packet drops is discussed to achieve stability, enhance the tracking, and improve [147]. Moreover, a Passivity-Based Packet Modulation (PBPM) is designed to handle packet losses that occur in the communication of signals over the communication channel.

Security challenges in BTSs reflect back to the operator reaction forces from the task being executed is discussed [148]. The cyber attacks are classified with regards to the objective component, knowledge of the system, and the purpose of the attack. Figure 6 shows all possible kind of cyebr attacks [148].

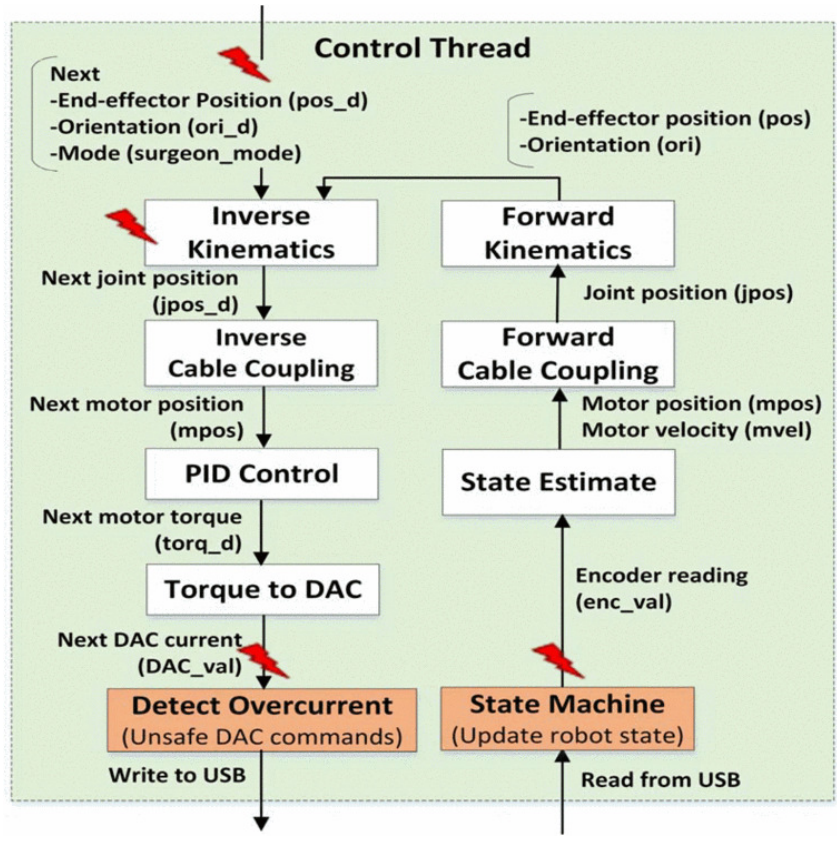

Figure 5. The kinematics chain in the RAVEN II control software

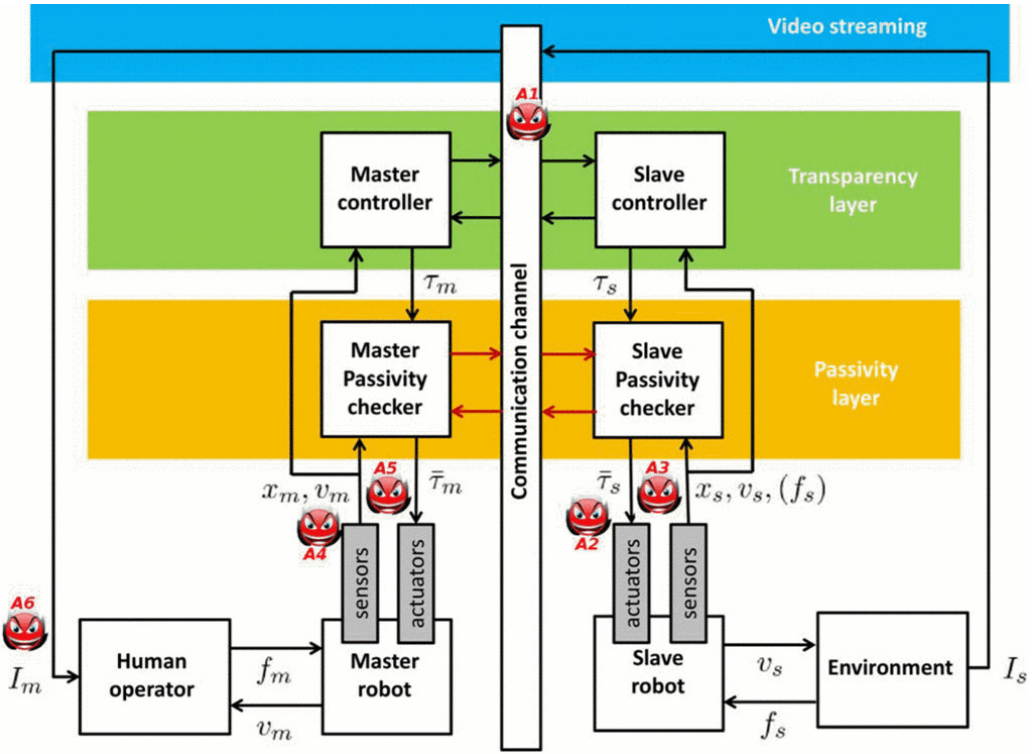

Figure 6. Two-layers BTS with potential vulnerabilities 
The attacks which aim to modify the content of signals on BTSs were discussed [149]. Initially, the vulnerability of BTSs to content modification attacks is considered, by which the attacker has the ability to change states i.e. the signals transmitted between the master and the slave sides. Then, a static malignant content modification attack (MCoMA) is defined such that to assure that the robot joint velocities are rendered unstable. After that, A safety mechanism is then defined to secure the system against static MCoMA. In the end, a dynamic subterfuge MCoMA that bypasses the safety mechanism is designed. Figure 7 shows the ability of the attacker to affect the signal between the master and the slave in several ways such as receiving, interpreting, manipulating, and transmitting the signals.

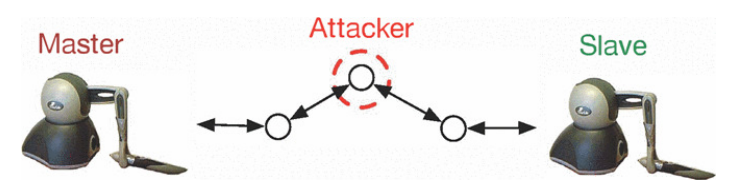

Figure 7. The capability of the attacker to capture one of the nodes in the BTS

False data injection attacks in BTSs are discussed, in which the attacker can insert false signal into the states being transmitted through the communication channel in the BTS [150]. a destabilizing false data injection attack (DFDIA) is created for demonstrating the vulnerability of the BTS and applied by experiments. A physics-based detection method including encoder and decoder is designed for detecting any FDI attack including the aforementioned DFDIA. For a BTS with a pair of nonlinear revolute robotic manipulators connected through a communication channel as presented in Figure 8. Now, let us ignore the external disturbances and friction, the dynamics of $n$-link master and slave robots are represented by (47):

$$
\begin{aligned}
M_{m}\left(q_{m}\right) \ddot{q}_{m}+C_{m}\left(q_{m}, \dot{q}_{m}\right) \dot{q}_{m} & =\tau_{h}+u_{m} \\
M_{s}\left(q_{s}\right) \ddot{q}_{s}+C_{s}\left(q_{s}, \dot{q}_{s}\right) \dot{q}_{s} & =u_{s}-\tau_{e} .
\end{aligned}
$$

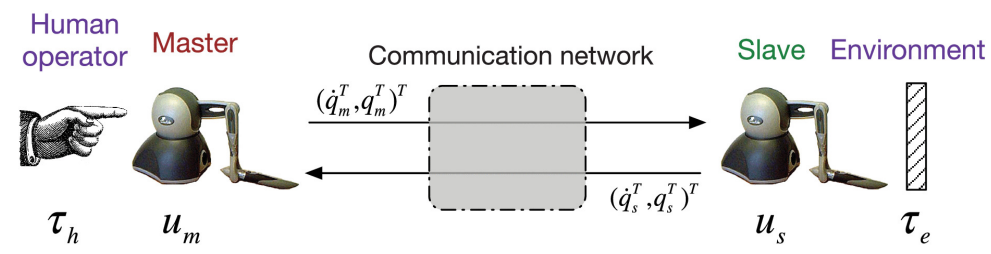

Figure 8. Example of BTS structure.

The subscript $i$ refer to both master and slave robots. Also, $\ddot{q}_{i}, \dot{q}_{i}, q_{i} \in \mathbb{R}^{n}$ are the angular acceleration, velocity, and position, respectively, $M_{i}\left(q_{i}\right) \in \mathbb{R}^{n \times n}$ is the inertia matrix, $C_{i}\left(q_{i}, \dot{q}_{i}\right) \in \mathbb{R}^{n \times n}$ is the centrifugal and Coriolis matrix, $G_{i}\left(q_{i}\right) \in \mathbb{R}^{n}$ is the gravitational torque, $\tau_{i} \in \mathbb{R}^{n}$ is the robot control input, and $\tau_{h}, \tau_{e} \in$ $\mathbb{R}^{n}$ are torques exerted by the human operator and the environment, respectively. When there is no attack $\left(\tilde{\dot{q}}_{i}=\dot{q}_{i}, \tilde{q}_{i}=q_{i}\right)$, the following PD-like controller can be adopted for BTS shown in (48):

$$
\begin{gathered}
u_{m}=-K_{d}\left(\dot{q}_{m}-\tilde{\dot{q}}_{s}\right)-K_{p}\left(q_{m}-\tilde{q}_{s}\right)-K_{d m} \dot{q}_{m} \\
u_{s}=-K_{d}\left(\dot{q}_{s}-\tilde{\dot{q}}_{m}\right)-K_{p}\left(q_{s}-\tilde{q}_{m}\right)-K_{d m} \dot{q}_{s}
\end{gathered}
$$

where $K_{d}, K_{p}, K_{d m} \in \mathbb{R}^{+}$.

For the BTOS with a PD-like control method represented in Figure 9, the attacker can affect the system by FDI attacks, which can intelligently replace the signals $\left(\dot{q}_{i}^{T}, q_{i}^{T}\right)^{T}$ being exchanged by the robots with $\left(\tilde{\dot{q}}_{i}^{T}, \tilde{q}_{i}^{T}\right)^{T}$ for $i=m, s$. For BTS, the DFDIA is discussed with respect to a specific form of storage function represented by (49):

$$
\begin{array}{r}
V=\frac{1}{2} \dot{q}_{s}^{T} M_{s} \dot{q}_{s}+\frac{1}{2} \dot{q}_{m}^{T} M_{m} \dot{q}_{m}+\frac{1}{2}\left(q_{s}-q_{m}\right)^{T} K_{p}\left(q_{s}-q_{m}\right) \\
-\int_{0}^{t} \dot{q}_{m}^{T} \tau_{h} d s+\beta_{h}+\int_{0}^{t} \dot{q}_{s}^{T} \tau_{e} d s+\beta_{e}
\end{array}
$$




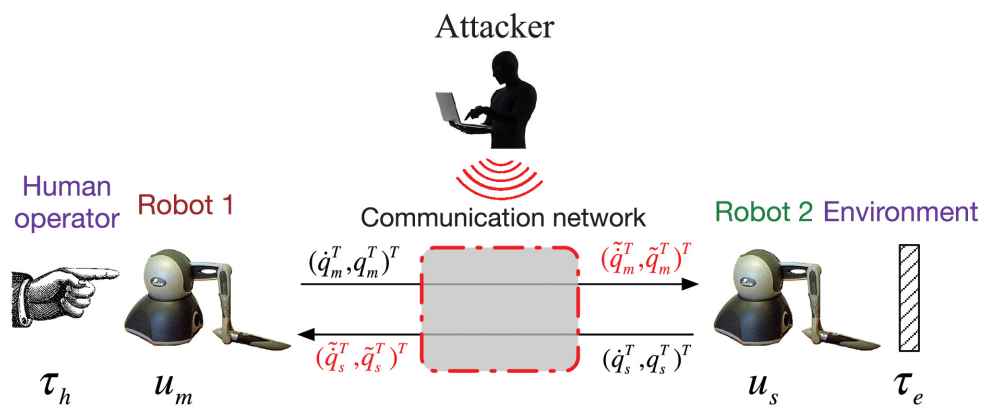

Figure 9. The formal definition of DFDIA can be given as follows

Theorem 1. In 2016, Dong, Gupta and Chopra [150] For a BTOS with dynamics (47) controlled by (48). A FDI attack that changes the states being transmitted between the robots of the BTSs as (50):

$$
\tilde{q}=q+\hat{q}=q+K q
$$

with $q=\left(\dot{q}_{s}^{T}, q_{s}^{T}, \dot{q}_{m}^{T}, q_{m}^{T}\right)^{T}, \tilde{q}=\left(\tilde{\dot{q}}_{s}^{T}, \tilde{q}_{s}^{T}, \tilde{\dot{q}}_{m}^{T}, \tilde{q}_{m}^{T}\right)^{T}$, and “attack gain" constant $K \in \mathbb{R}^{4 n \times 4 n}$ is a DFDIA with respect to the storage function $V$ in (49) if and only if: 1) $P:=A+B K \geq 0$ and 2) the set $\boldsymbol{S} \backslash \boldsymbol{E}$ is not positively invariant, where $\boldsymbol{S}:=\left\{q \in \mathbb{R}^{4 n} \mid q^{T} P q=0\right\}$

$$
\begin{aligned}
A & =\left[\begin{array}{cccc}
-\left(K_{d m}+K_{d}\right) I_{n} & 0_{n} & K_{d} I_{n} & 0_{n} \\
0_{n} & 0_{n} & 0_{n} & 0_{n} \\
K_{d} I_{n} & 0_{n} & -\left(K_{d m}+K_{d}\right) I_{n} & 0_{n} \\
0_{n} & 0_{n} & 0_{n} & 0_{n}
\end{array}\right] \\
B & =\left[\begin{array}{cccc}
0_{n} & 0_{n} & K_{d} I_{n} & K_{p} I_{n} \\
0_{n} & 0_{n} & 0_{n} & 0_{n} \\
K_{d} I_{n} & K_{p} I_{n} & 0_{n} & 0_{n} \\
0_{n} & 0_{n} & 0_{n} & 0_{n}
\end{array}\right] .
\end{aligned}
$$

For each transmission sequence $k_{i}$, the encoding and decoding factors are updated with the same dynamics $f_{1}, f_{2}$ as (51)-(55):

$$
\begin{aligned}
& { }^{i} \lambda_{i 1}^{k_{i}}={ }^{i} \lambda_{i 1}^{k_{i}-1}+T_{i} f_{1}\left({ }^{i} \lambda_{i 1}^{k_{i}-1}, k_{i}\right) \\
& { }^{i} \lambda_{i 2}^{k_{i}}={ }^{i} \lambda_{i 2}^{k_{i}-1}+T_{i} f_{2}\left({ }^{i} \lambda_{i 2}^{k_{i}-1}, k_{i}\right)
\end{aligned}
$$

and

$$
\begin{aligned}
& { }^{i} \lambda_{j 1}^{k_{i}}={ }^{i} \lambda_{j 1}^{k_{i}-1}+T_{i} f_{1}\left({ }^{i} \lambda_{j 1}^{k_{i}-1}, k_{i}\right) \\
& { }^{i} \lambda_{j 2}^{k_{i}}={ }^{i} \lambda_{j 2}^{k_{i}-1}+T_{i} f_{2}\left({ }^{i} \lambda^{k_{2}-1}, k_{i}\right)
\end{aligned}
$$

Also,

$$
\begin{gathered}
r_{i 1}^{k_{i}}=\dot{q}_{i}^{k_{i}}+{ }^{i} \lambda_{1}^{k_{i}} q_{i}^{k_{i}}, \quad r_{i 2}^{k_{i}}=\dot{q}_{i}^{k_{i}}+{ }^{i} \lambda_{2}^{k_{i}} q_{i}^{k_{i}} . \\
q_{i}^{k_{i}}={ }^{i} a^{k_{i}}\left(r_{i 1}^{k_{i}}-r_{i 2}^{k_{i}}\right), \quad \dot{q}_{i}^{k_{i}}={ }^{i} b^{k_{i}} r_{i 1}^{k_{i}}-{ }^{i} c^{k_{i}} r_{i 2}^{k_{i}} \\
\frac{q_{i}^{k_{i}}-q_{i}^{k_{i}-1}}{T_{i}}=\dot{q}_{i}^{k_{i}} .
\end{gathered}
$$

The detection approach is summarized as shown in Algorithm 1. 


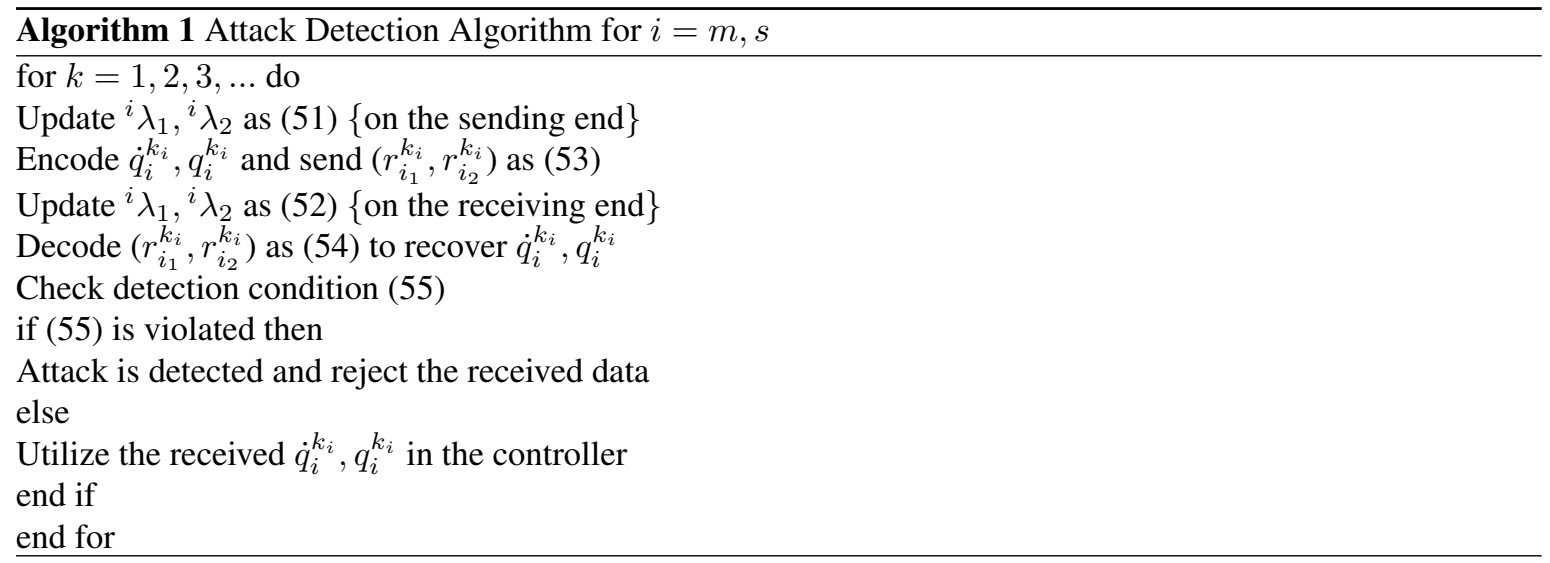

\section{CONCLUSIONS}

The teleoperation system is often composed of a human operator, a local master manipulator, and a remote slave manipulator that are connected by a communication network. This paper presents a comprehensive survey on feedback control design for the BTSs in both nominal situations and in the presence of cyber-attacks. The main objective of the discussed methods is to achieve the stability of a delayed bilateral teleoperation system in the presence of several kinds of cyber attacks. In this paper, a comprehensive survey on control systems for BTSs under cyber-attacks is discussed. Finally, we discuss the current and future problems in this field.

Several security issues of BTSs controllers have been discussed in the literature. However, many problems have not been addressed yet. some of these problems are: control framework encompasses the issue of time-varying delays and transparency in the tasks-space teleoperation system. Also, the problem of the dead zone in robotic arms and the use of the neural network for uncertainties. For the cyber attacks, the problems of considering several attacks in BTSs especially simultaneous cyber-attacks have not been discussed in the literature. Another promising research area is the implementation of deep learning and artificial intelligence in the detection and control of BTSs.

\section{ACKNOWLEDGMENTS}

This work is supported by the deanship of scientific research (DSR) at KFUPM through distinguished professorship award project no. DUP19106.

\section{REFERENCES}

[1] P. F. Hokayem and M. W. Spong, "Bilateral teleoperation: An historical survey," Automatica, vol. 42, no. 12, pp. 2035- 2057, 2006, doi: 10.1016/j.automatica.2006.06.027.

[2] E. Nuño, L. Basañez and R. Ortega, "Passivity-based control for bilateral teleoperation: A tutorial," Automatica, vol. 47, no. 3, pp. 485-495, 2011, doi: 10.1016/j.automatica.2011.01.004.

[3] M. Ferre, M. Buss, R. Aracil, C. Melchiorri, C. Balaguer, Advances in Telerobotics, German: Springer, 2007.

[4] F. Hashemzadeh, I. Hassanzadeh and M. Tavakoli, "Teleoperation in the presence of varying time delays and sandwich linearity in actuators," Automatica, vol. 49, no. 9, pp. 2813-2821,2013, doi: 10.1016/j.automatica.2013.05.012.

[5] S. H. Ahn, B. S. Park and J. S. Yoon, "A teleoperation position control for 2-DOF manipulators with control input saturation," ISIE 2001. 2001 IEEE International Symposium on Industrial Electronics Proceedings (Cat. No.01TH8570), 2001, pp. 1520-1525 vol.3, doi: 10.1109/ISIE.2001.931931.

[6] K. . -B. Sim, K. . -S. Byun and F. Harashima, "Internet-Based Teleoperation of an Intelligent Robot With Optimal Two-Layer Fuzzy Controller,' in IEEE Transactions on Industrial Electronics, vol. 53, no. 4, pp. 1362-1372, June 2006, doi: 10.1109/TIE.2006.878295.

[7] Z. Li, L. Ding, H. Gao, G. Duan and C. Su, "Trilateral Teleoperation of Adaptive Fuzzy Force/Motion 
Control for Nonlinear Teleoperators With Communication Random Delays," in IEEE Transactions on Fuzzy Systems, vol. 21, no. 4, pp. 610-624, Aug. 2013, doi: 10.1109/TFUZZ.2012.2224116.

[8] Z. Wen-Hong and S. E. Salcudean, "Stability guaranteed teleoperation: an adaptive motion/force control approach," in IEEE Transactions on Automatic Control, vol. 45, no. 11, pp. 1951-1969, Nov. 2000, doi: $10.1109 / 9.887620$

[9] K. Byeong-Yeon and A. Hyo-Sung, "A design of bilateral teleoperation systems using composite adaptive controller," Control Engineering Practice, vol. 12, no. 12, pp. 1641-1652, 2013, doi: 10.1016/j.conengprac.2013.08.013.

[10] E. Nuño, R. Ortega and L. Basañez, "An adaptive controller for nonlinear teleoperators," Automatica, vol. 46, no. 1, pp. 155-159, 2010, doi: 10.1016/j.automatica.2009.10.026.

[11] Z. Li, Y. Xia and X. Cao, "Adaptive Control Of Bilateral Teleoperationwith Unsymmetrical TimeVarying Delays," International Journal of Innovative Computing, Information and Control, vol. 9, no. 2, pp. 653-767, 2013.

[12] Nikhil Chopra, Mark W. Spong and Rogelio Lozano, "Synchronization of bilateral teleoperators with time delay," Automatica, vol. 44, no. 8, pp. 2142-2148, 2008, doi:10.1016/j.automatica.2007.12.002.

[13] E. Nuño, R. Ortega, L. Basañez and D. Hill, "Synchronization of Networks of Nonidentical EulerLagrange Systems With Uncertain Parameters and Communication Delays," in IEEE Transactions on Automatic Control, vol. 56, no. 4, pp. 935-941, April 2011, doi: 10.1109/TAC.2010.2103415.

[14] E. NuÑo, R. Ortega, N. Barabanov and L. BasaÑez, "A Globally Stable PD Controller for Bilateral Teleoperators," in IEEE Transactions on Robotics, vol. 24, no. 3, pp. 753-758, June 2008, doi: 10.1109/TRO.2008.921565.

[15] Di-Hua Zhai and Yuanqing Xia, "Robust saturation-based control of bilateral teleoperation without velocity measurements," International Journal of Robust and Nonlinear Control,vol. 25, no. 15, pp. 2582 2607, 2015, doi:10.1002/rnc.3214.

[16] Y. Yang, C. Hua and X. Guan, "Adaptive Fuzzy Finite-Time Coordination Control for Networked Nonlinear Bilateral Teleoperation System," in IEEE Transactions on Fuzzy Systems, vol. 22, no. 3, pp. 631-641, June 2014, doi: 10.1109/TFUZZ.2013.2269694.

[17] Jong Hyeon Park and Hyun Chul Cho, "Sliding-mode controller for bilateral teleoperation with varying time delay," 1999 IEEE/ASME International Conference on Advanced Intelligent Mechatronics (Cat. No.99TH8399), 1999, pp. 311-316, doi: 10.1109/AIM.1999.803184.

[18] Y. Kang, Z. Li, W. Shang, and H. Xi, "Motion synchronisation of bilateral teleoperation systems with mode-dependent time-varying communication delays," IET Control Theory \& Applications, vol. 4, no. 10, pp. 2129-2140, 2010, doi: 10.1049/iet-cta.2009.0638.

[19] L. Ma and K. Schilling, "Survey on bilateral teleoperation of mobile robots," In Proceedings of the 13th IASTED International Conference on Robotics and Applications (RA '07), 2007, pp. 489-494.

[20] C. Passenberg, A. Peer and M. Buss, "A survey of environment-, operator-, and task-adapted controllers for teleoperation systems," Mechatronics, vol. 20, no. 7, pp. 787-801, 2010, doi: 10.1016/j.mechatronics.2010.04.005.

[21] G. V. A. G. A. Perera and A. M. H. S. Abeykoon, "Review on bilateral teleoperation with force, position, power and impedance scaling," 7th International Conference on Information and Automation for Sustainability, 2014, pp. 1-7, doi: 10.1109/ICIAFS.2014.7069636.

[22] E. Delgado, M. Díaz-Cacho and A. Barreiro, "Stability of Teleoperation Systems for Time-Varying Delays by Neutral LMI Techniques," Mathematical Problems in Engineering, vol. 2012, p. 467629, 2012, doi: 10.1155/2012/467629.

[23] P. Arcara and C. Melchiorri, "Control schemes for teleoperation with time delay: A comparative study," Robotics and Autonomous Systems, vol. 38, no. 1, pp. 49-64, 2002, doi: 10.1016/S09218890(01)00164-6.

[24] R. J. Anderson and M. W. Spong, "Bilateral control of teleoperators with time delay," in IEEE Transactions on Automatic Control, vol. 34, no. 5, pp. 494-501, May 1989, doi: 10.1109/9.24201.

[25] O. Kostyukova, F. P. Vista IV and K. T. Chong, "Design of feedforward and feedback position control for passive bilateral teleoperation with delays," ISA Transactions, vol. 85, pp. 200-213, 2019.

[26] J. Zhu, X. He and W. Gueaieb, "Trends in the Control Schemes for Bilateral Teleoperation with Time Delay," in Autonomous and Intelligent Systems, Kamel M., Karray F., Gueaieb W., Khamis A. Ed., Germany: Springer, 2011, doi: 10.1007/978-3-642-21538-4_15. 
[27] C. Hua and P. X. Liu, "Delay-dependent stability analysis of teleoperation systems with unsymmetric time-varying delays," 2009 IEEE International Conference on Robotics and Automation, 2009, pp. 11461151, doi: 10.1109/ROBOT.2009.5152630.

[28] C. Hua, Y. Yang, X. Yang, adn X. Guan, "Stability Analysis of Teleoperation Systems with Asymmetric Time-Varying Delays," in Analysis and Design for Networked Teleoperation System, Singapore: Springer, 2019.

[29] Y. Yuan2018Y. Yuan, Y. Wang, H. Yang and C. Sun, ”Adaptive Force Reflecting Control for Bilateral Teleoperation System Under Asymmetric Time-Varying Delays," 2018 37th Chinese Control Conference (CCC), 2018, pp. 687-691, doi: 10.23919/ChiCC.2018.8482540.

[30] M. V. de Lima, L. A. Mozelli, A. A. Neto, and F. O. Souza, "A simple algebraic criterion for stability of Bilateral Teleoperation Systems under time-varying delays," Mechanical Systems and Signal Processing, vol. 137, p. 106217, 2020, doi: 10.1016/j.ymssp.2019.06.035.

[31] C. Hua and X. P. Liu, "Delay-Dependent Stability Criteria of Teleoperation Systems With Asymmetric Time-Varying Delays," in IEEE Transactions on Robotics, vol. 26, no. 5, pp. 925-932, Oct. 2010, doi: 10.1109/TRO.2010.2053736.

[32] C. Hua and P. X. Liu, "Convergence Analysis of Teleoperation Systems With Unsymmetric TimeVarying Delays," in IEEE Transactions on Circuits and Systems II: Express Briefs, vol. 56, no. 3, pp. 240-244, March 2009, doi: 10.1109/TCSII.2009.2015364.

[33] A. A. Ghavifekr, A. R. Ghiasi, and M. A. Badamchizadeh, "Discrete-time control of bilateral teleoperation systems: a review," Robotica, vol. 36, no. 4, pp. 552-569, 2018, doi: 10.1017/S0263574717000583.

[34] P. M. Kebria, H. Abdi, M. M. Dalvand, A. Khosravi and S. Nahavandi, "Control Methods for InternetBased Teleoperation Systems: A Review," in IEEE Transactions on Human-Machine Systems, vol. 49, no. 1, pp. 32-46, Feb. 2019, doi: 10.1109/THMS.2018.2878815.

[35] R. Uddin and J. Ryu, "Predictive control approaches for bilateral teleoperation," Annual Reviews in Control, vol. 42, pp. 82-99, 2016, doi: 10.1016/j.arcontrol.2016.09.003.

[36] L. Yen-Chen and N. Chopra, "Control of semi-autonomous teleoperation system with time delays," Automatica, vol. 49, no. 6, pp. 1553-1565, 2013, doi: 10.1016/j.automatica.2013.02.009.

[37] H. Wang, "Passivity based synchronization for networked robotic systems with uncertain kinematics and dynamics," Automatica, vol. 49, no. 3, pp. 755-761, 2013, doi: 10.1016/j.automatica.2012.11.003.

[38] H. Du, "H state-feedback control of bilateral teleoperation systems with asymmetric time-varying delays," IET Control Theory \& Applications, vol. 7, no. 4, pp. 596-605, 2013, doi: 10.1049/ietcta.2011.0643.

[39] E. W. Tunstel Jr., et al., "Recent enhancements to mobile bimanual robotic teleoperation with insight toward improving operator control," Johns Hopkins APL technical digest., vol. 32, no. 3, pp. 584-594, 2013.

[40] D. Sun, F. Naghdy and H. Du, "Application of wave-variable control to bilateral teleoperation systems: A survey," Annual Reviews in Control, vol. 38, no. 1, pp. 12-31, 2014, doi: 10.1016/j.arcontrol.2014.03.002.

[41] L. Chan, F. Naghdy and D. Stirling, "Application of Adaptive Controllers in Teleoperation Systems: A Survey," in IEEE Transactions on Human-Machine Systems, vol. 44, no. 3, pp. 337-352, June 2014, doi: 10.1109/THMS.2014.2303983.

[42] F. Hashemzadeh, M. Sharifi and M. Tavakoli, "Nonlinear trilateral teleoperation stability analysis subjected to time-varying delays," Control Engineering Practice, vol. 56, pp. 123-135, 2016, doi: 10.1016/j.conengprac.2016.08.004.

[43] E. Slawiñski, S. García, L. Salinas, and V. Mut, "PD-like controller with impedance for delayed bilateral teleoperation of mobile robots," Robotica, vol. 34, no. 9, pp. 2151-2161, 2016, doi: $10.1017 / \mathrm{S} 0263574715000223$.

[44] D. Sun, F. Naghdy and H. Du, "A novel approach for stability and transparency control of nonlinear bilateral teleoperation system with time delays," Control Engineering Practice, vol. 47, pp. 14-27, 2016, doi: 10.1016/j.conengprac.2015.11.003.

[45] A. Haddadi, K. Razi and K. Hashtrudi-Zaad, "Operator Dynamics Consideration for Less Conservative Coupled Stability Condition in Bilateral Teleoperation," in IEEE/ASME Transactions on Mechatronics, vol. 20, no. 5, pp. 2463-2475, Oct. 2015, doi: 10.1109/TMECH.2014.2385637.

[46] J. M. Daly and D. W. L. Wang, ”Time-Delayed Output Feedback Bilateral Teleoperation With Force 
Estimation for $n$-DOF Nonlinear Manipulators," in IEEE Transactions on Control Systems Technology, vol. 22, no. 1, pp. 299-306, Jan. 2014, doi: 10.1109/TCST.2013.2242329.

[47] A. M. Ousaid, D. S. Haliyo, S. Régnier and V. Hayward, ”A Stable and Transparent Microscale Force Feedback Teleoperation System," in IEEE/ASME Transactions on Mechatronics, vol. 20, no. 5, pp. 2593-2603, Oct. 2015, doi: 10.1109/TMECH.2015.2423092.

[48] H. Amini, S. M. Rezaei, Ahmed A. D. Sarhan, J. Akbari, and N. A. Mardi, 'Transparency Improvement by External Force Estimation in a Time-Delayed Nonlinear Bilateral Teleoperation System," J. Dyn. Sys., Meas., Control., vol. 137, no. 5, p. 0151013, 2015, doi: 10.1115/1.4029077.

[49] V. Chawda and M. K. O’Malley, "Position Synchronization in Bilateral Teleoperation Under TimeVarying Communication Delays," in IEEE/ASME Transactions on Mechatronics, vol. 20, no. 1, pp. 245-253, Feb. 2015, doi: 10.1109/TMECH.2014.2317946.

[50] S. Islam, P. X. Liu and A. El Saddik, "New stability and tracking criteria for a class of bilateral teleoperation systems," Information Sciences, vol. 278, pp. 868-882, 2014, doi: 10.1016/j.ins.2014.03.100.

[51] R. Sepulchre, M. Jankovic and P. V. Kokotovic, Constructive Nonlinear Control, Springer, 1997.

[52] M. W. Spong, S. Hutchinson and M. Vidyasagar, Robot Modeling and Control, Wiley, 2005.

[53] R. Ortega, Z. P. Jiang and D. J. Hill, "Passivity-based control of nonlinear systems: a tutorial," Proceedings of the 1997 American Control Conference (Cat. No.97CH36041), 1997, pp. 2633-2637 vol.5, doi: 10.1109/ACC.1997.611933.

[54] G. Niemeyer and J. -. E. Slotine, "Using wave variables for system analysis and robot control," Proceedings of International Conference on Robotics and Automation, 1997, pp. 1619-1625 vol.2, doi: 10.1109/ROBOT.1997.614372.

[55] E. Kamrani, "Real-Time Internet-Based Teleoperation," Intelligent Control and Automation, vol. 3, no. 4, pp. 356-375, 2012, doi: 10.4236/ica.2012.34041.

[56] M. Franken, S. Stramigioli, S. Misra, C. Secchi and A. Macchelli, "Bilateral Telemanipulation With Time Delays: A Two-Layer Approach Combining Passivity and Transparency," in IEEE Transactions on Robotics, vol. 27, no. 4, pp. 741-756, Aug. 2011, doi: 10.1109/TRO.2011.2142430.

[57] K. Hertkorn, T. Hulin, P. Kremer, C. Preusche and G. Hirzinger, "Time Domain Passivity Control for multi-degree of freedom haptic devices with time delay," 2010 IEEE International Conference on Robotics and Automation, 2010, pp. 1313-1319, doi: 10.1109/ROBOT.2010.5509148.

[58] J. Artigas, J. Ryu and C. Preusche, "Position drift compensation in time domain passivity based teleoperation," 2010 IEEE/RSJ International Conference on Intelligent Robots and Systems, 2010, pp. 42504256, doi: 10.1109/IROS.2010.5652691.

[59] C. Ott, J. Artigas and C. Preusche, ”Subspace-oriented energy distribution for the Time Domain Passivity Approach," 2011 IEEE/RSJ International Conference on Intelligent Robots and Systems, 2011, pp. 665671, doi: 10.1109/IROS.2011.6094697.

[60] M. Franken, B. Willaert, S. Misra and S. Stramigioli, "Bilateral telemanipulation: Improving the complementarity of the frequency- and time-domain passivity approaches," 2011 IEEE International Conference on Robotics and Automation, 2011, pp. 2104-2110, doi: 10.1109/ICRA.2011.5979969.

[61] J. Ware and Y.-J. Pan, "Realisation of a bilaterally teleoperated robotic vehicle platform with passivity control," IET Control Theory \& Applications, vol. 5, no, 8, pp. 952-962, 2011, doi: https://doi.org/10.1049/iet-cta.2010.0265.

[62] S. F. Atashzar, M. Shahbazi, M. Tavakoli and R. V. Patel, "A Passivity-Based Approach for Stable Patient-Robot Interaction in Haptics-Enabled Rehabilitation Systems: Modulated Time-Domain Passivity Control," in IEEE Transactions on Control Systems Technology, vol. 25, no. 3, pp. 991-1006, May 2017, doi: 10.1109/TCST.2016.2594584.

[63] L. Weihua, L. Zhen, G. Haibo, Z. Xuefeng, and M. Tavakoli, "Stable kinematic teleoperation of wheeled mobile robots with slippage using time-domain passivity control," Mechatronics, vol. 39, pp. 196-203, 2016, doi: 10.1016/j.mechatronics.2016.05.005.

[64] U. Ahmad and Y. Pan, "Switching time domain passivity control for multilateral teleoperation systems under time varying delays," 2016 IEEE 55th Conference on Decision and Control (CDC), 2016, pp. 1429-1434, doi: 10.1109/CDC.2016.7798467.

[65] B. H. Jafari and M. W. Spong, "Passivity-based switching control in teleoperation systems with timevarying communication delay," 2017 American Control Conference (ACC), 2017, pp. 5469-5475, doi: 10.23919/ACC.2017.7963805. 
[66] D. Sun, F. Naghdy and H. Du, "Neural Network-Based Passivity Control of Teleoperation System Under Time-Varying Delays," in IEEE Transactions on Cybernetics, vol. 47, no. 7, pp. 1666-1680, July 2017, doi: 10.1109/TCYB.2016.2554630.

[67] D. Sun, F. Naghdy and H. Du, "Wave-Variable-Based Passivity Control of Four-Channel Nonlinear Bilateral Teleoperation System Under Time Delays," in IEEE/ASME Transactions on Mechatronics, vol. 21, no. 1, pp. 238-253, Feb. 2016, doi: 10.1109/TMECH.2015.2442586.

[68] Z. Chen, F. Huang, W. Sun and W. Song, "An Improved Wave-Variable Based Four-Channel Control Design in Bilateral Teleoperation System for Time-Delay Compensation,” in IEEE Access, vol. 6, pp. 12848-12857, 2018, doi: 10.1109/ACCESS.2018.2805782.

[69] M. Shahbazi, S. F. Atashzar, M. Tavakoli and R. V. Patel, "Position-Force Domain Passivity of the Human Arm in Telerobotic Systems," in IEEE/ASME Transactions on Mechatronics, vol. 23, no. 2, pp. 552-562, April 2018, doi: 10.1109/TMECH.2018.2793877.

[70] J. -H. Ryu, Q. Ha-Van and A. Jafari, ”Multilateral Teleoperation Over Communication Time Delay Using the Time-Domain Passivity Approach," in IEEE Transactions on Control Systems Technology, vol. 28, no. 6, pp. 2705-2712, Nov. 2020, doi: 10.1109/TCST.2019.2948126.

[71] D. B. Venkateswaran and Z. Qu, "A Passivity-Shortage Based Control Design for Teleoperation With Time-Varying Delays," in IEEE Robotics and Automation Letters, vol. 5, no. 3, pp. 4070-4077, July 2020, doi: 10.1109/LRA.2020.2988142.

[72] G. Niemeyer and J. -. E. Slotine, "Stable adaptive teleoperation,” in IEEE Journal of Oceanic Engineering, vol. 16, no. 1, pp. 152-162, Jan. 1991, doi: 10.1109/48.64895.

[73] E. Mujčić, A. Mujčić and S. Pajazetović, "Internet-based teleoperation using wave variables and correction of position error," 2016 International Conference on Smart Systems and Technologies (SST), 2016, pp. 219-224, doi: 10.1109/SST.2016.7765663.

[74] Y. Yokokohji, T. Tsujioka and T. Yoshikawa, "Bilateral control with time-varying delay including communication blackout," Proceedings 10th Symposium on Haptic Interfaces for Virtual Environment and Teleoperator Systems. HAPTICS 2002, 2002, pp. 285-292, doi: 10.1109/HAPTIC.2002.998970.

[75] D. Sun, F. Naghdy, and H. Du, "Transparent four-channel bilateral control architecture using modified wave variable controllers under time delays," Robotica, vol. 34, no. 4, pp. 859-875, 2016, doi: 10.1017/S026357471400191X.

[76] H. Li and K. Kawashima, "Achieving Stable Tracking in Wave-Variable-Based Teleoperation," in IEEE/ASME Transactions on Mechatronics, vol. 19, no. 5, pp. 1574-1582, Oct. 2014, doi: 10.1109/TMECH.2013.2289076.

[77] N. D'Amore and D. L. Akin, "Transparency and Tuning of Wave-Based Bilateral Teleoperation Systems," in IEEE Robotics and Automation Letters, vol. 2, no. 1, pp. 321-328, Jan. 2017, doi: 10.1109/LRA.2016.2606659.

[78] S. Munir and W. J. Book, "Wave-based teleoperation with prediction," Proceedings of the 2001 American Control Conference. (Cat. No.01CH37148), 2001, pp. 4605-4611 vol.6, doi: 10.1109/ACC.2001.945706.

[79] C. Benedetti, M. Franchini and P. Fiorini, "Stable tracking in variable time-delay teleoperation," Proceedings 2001 IEEE/RSJ International Conference on Intelligent Robots and Systems. Expanding the Societal Role of Robotics in the the Next Millennium (Cat. No.01CH37180), 2001, pp. 2252-2257 vol.4, doi: 10.1109/IROS.2001.976405.

[80] F. Ferraguti, C. Fantuzzi and C. Secchi, "Optimizing the use of power in wave based bilateral teleoperation," 2016 IEEE/RSJ International Conference on Intelligent Robots and Systems (IROS), 2016, pp. 1469-1474, doi: 10.1109/IROS.2016.7759239.

[81] D. Zhai and Y. Xia, "Adaptive Control for Teleoperation System With Varying Time Delays and Input Saturation Constraints," in IEEE Transactions on Industrial Electronics, vol. 63, no. 11, pp. 6921-6929, Nov. 2016, doi: 10.1109/TIE.2016.2583199.

[82] D. Zhai and Y. Xia, "Adaptive finite-time control for nonlinear teleoperation systems with asymmetric time-varying delays," International Journal of Robust and Nonlinear Control, vol. 26, no. 12,pp. 2586-2607, 2016, doi: 10.1002/rnc.3462.

[83] D. Zhai and Y. Xia, "Adaptive Fuzzy Control of Multilateral Asymmetric Teleoperation for Coordinated Multiple Mobile Manipulators," in IEEE Transactions on Fuzzy Systems, vol. 24, no. 1, pp. 57-70, Feb. 2016, doi: 10.1109/TFUZZ.2015.2426215. 
[84] Z. Li, Y. Xia and F. Sun, "Adaptive Fuzzy Control for Multilateral Cooperative Teleoperation of Multiple Robotic Manipulators Under Random Network-Induced Delays," in IEEE Transactions on Fuzzy Systems, vol. 22, no. 2, pp. 437-450, April 2014, doi: 10.1109/TFUZZ.2013.2260550.

[85] Y. Yang, C. Hua and X. Guan, "Adaptive Fuzzy Finite-Time Coordination Control for Networked Nonlinear Bilateral Teleoperation System," in IEEE Transactions on Fuzzy Systems, vol. 22, no. 3, pp. 631-641, June 2014, doi: 10.1109/TFUZZ.2013.2269694.

[86] Z. Chen, Y.-J. Pan, and J. Gu, "Integrated adaptive robust control for multilateral teleoperation systems under arbitrary time delays," International Journal of Robust and Nonlinear Control, vol. 26, no. 12, pp. 2708-2728, 2016, doi: 10.1002/rnc.3472.

[87] C. Hua, Y. Yang and P. X. Liu, "Output-Feedback Adaptive Control of Networked Teleoperation System With Time-Varying Delay and Bounded Inputs," in IEEE/ASME Transactions on Mechatronics, vol. 20, no. 5, pp. 2009-2020, Oct. 2015, doi: 10.1109/TMECH.2014.2359969.

[88] J. Corredor, J. Sofrony and A. Peer, "Decision-Making Model for Adaptive Impedance Control of Teleoperation Systems," in IEEE Transactions on Haptics, vol. 10, no. 1, pp. 5-16, 1 Jan.-March 2017, doi: 10.1109/TOH.2016.2581807.

[89] Z. Li and C. Su, "Neural-Adaptive Control of Single-Master-Multiple-Slaves Teleoperation for Coordinated Multiple Mobile Manipulators With Time-Varying Communication Delays and Input Uncertainties," in IEEE Transactions on Neural Networks and Learning Systems, vol. 24, no. 9, pp. 1400-1413, Sept. 2013, doi: 10.1109/TNNLS.2013.2258681.

[90] Y. Liu and M. Khong, "Adaptive Control for Nonlinear Teleoperators With Uncertain Kinematics and Dynamics," in IEEE/ASME Transactions on Mechatronics, vol. 20, no. 5, pp. 2550-2562, Oct. 2015, doi: 10.1109/TMECH.2015.2388555.

[91] A. Hace and M. Franc, "Pseudo-Sensorless High-Performance Bilateral Teleoperation by Sliding-Mode Control and FPGA," in IEEE/ASME Transactions on Mechatronics, vol. 19, no. 1, pp. 384-393, Feb. 2014, doi: 10.1109/TMECH.2013.2241778.

[92] M. Azadegan, S. Ozgoli and H. R. Taghirad, "Delay-independent robust stability analysis of teleoperation," 2011 Chinese Control and Decision Conference (CCDC), 2011, pp. 4129-4133, doi: 10.1109/CCDC.2011.5968949.

[93] A. Roushandel, A. Khosravi and A. Alfi, "Bilateral control of teleoperation systems via robust PID controllers based on LMI," The 3rd International Conference on Control, Instrumentation, and Automation, 2013, pp. 16-21, doi: 10.1109/ICCIAutom.2013.6912802.

[94] M. Salimifar, H. D. Taghirad and B. Fallahi, "Formulation of transparency in bilateral teleoperation systems: A robust approach," The 3rd International Conference on Control, Instrumentation, and $\mathrm{Au}$ tomation, 2013, pp. 22-27, doi: 10.1109/ICCIAutom.2013.6912803.

[95] Z. Chen, Y.-J. Pan, and J. Gu, "A novel adaptive robust control architecture for bilateral teleoperation systems under time-varying delays," International Journal of Robust and Non-linear Control, vol. 25, no. 17, pp. 3349-3366, 2015, doi: 10.1002/rnc.3267.

[96] C. A. López Martínez, R. van de Molengraft, S. Weiland and M. Steinbuch, "Switching Robust Control for Bilateral Teleoperation," in IEEE Transactions on Control Systems Technology, vol. 24, no. 1, pp. 172-188, Jan. 2016, doi: 10.1109/TCST.2015.2422795.

[97] H. Amini, V. Dabbagh, S. Rezaei, M. Zareinejad, N. Mardi, and A. A. Sarhan, "Robust control-based linear bilateral teleoperation system without force sensor,' Journal of the Brazilian Society of Mechanical Sciences and Engineering, vol. 37, pp. 579-587, 2015, doi: 10.1007/s40430-014-0207-2.

[98] B. Jing, J. Na, G. Gao and C. Yang, ”Robust adaptive control for bilateral teleoperation systems with guaranteed parameter estimation," 2016 International Conference on Advanced Robotics and Mechatronics (ICARM), 2016, pp. 32-37, doi: 10.1109/ICARM.2016.7606890.

[99] Y. Yang, C. Hua and X. Guan, "Finite Time Control Design for Bilateral Teleoperation System With Position Synchronization Error Constrained," in IEEE Transactions on Cybernetics, vol. 46, no. 3, pp. 609-619, March 2016, doi: 10.1109/TCYB.2015.2410785.

[100] Y. Liu, "Robust synchronisation of networked Lagrangian systems and its applications to multi-robot teleoperation," IET Control Theory \& Applications, vol. 9, no. 1, pp. 129-139,2 015, doi: 10.1049/ietcta.2013.0914.

[101] D. Zhai and Y. Xia, "Finite-Time Control of Teleoperation Systems With Input Saturation and Varying Time Delays," in IEEE Transactions on Systems, Man, and Cybernetics: Systems, vol. 47, no. 7, pp. 
1522-1534, July 2017, doi: 10.1109/TSMC.2016.2631601.

[102] Y. C. Liu, M. H. Khong, and T.-W. Ou, "Nonlinear bilateral teleoperators with noncollocated remote controller over delayed network," Mechatronics, vol. 45, pp.25-36, 2017, doi: 10.1016/j.mechatronics.2017.05.005.

[103] F. Lewis, A. Yesildirak, and S. Jagannatha, Neural Network Control Of Robot Manipulators And NonLinear Systems, CRC Press, 1998.

[104] H. Wang, P. X. Liu and S. Liu, "Adaptive Neural Synchronization Control for Bilateral Teleoperation Systems With Time Delay and Backlash-Like Hysteresis," in IEEE Transactions on Cybernetics, vol. 47, no. 10, pp. 3018-3026, Oct. 2017, doi: 10.1109/TCYB.2016.2644656.

[105] Z. Li, Y. Xia, D. Wang, D. Zhai, C. Su and X. Zhao, "Neural Network-Based Control of Networked Trilateral Teleoperation With Geometrically Unknown Constraints," in IEEE Transactions on Cybernetics, vol. 46, no. 5, pp. 1051-1064, May 2016, doi: 10.1109/TCYB.2015.2422785.

[106] L. Hongyan, W. Hong and G. Chao, "Internet Time-delay Prediction Based on Autoregressive and Neural Network Model," 2006 International Conference on Communications, Circuits and Systems, 2006, pp. 1758-1761, doi: 10.1109/ICCCAS.2006.285014.

[107] T. Auld, A. W. Moore and S. F. Gull, "Bayesian Neural Networks for Internet Traffic Classification," in IEEE Transactions on Neural Networks, vol. 18, no. 1, pp. 223-239, Jan. 2007, doi: 10.1109/TNN.2006.883010.

[108] S. Belhaj and M. Tagina, "Modeling and prediction of the internet end-to-end delay using recurrent neural networks," J. Networks, vol. 4, no. 6, pp. 528-535, 2009.

[109] M. M. Mirzaei, K. Mizanian and M. Rezaeian, "Modeling of self-similar network traffic using artificial neural networks," 2014 4th International Conference on Computer and Knowledge Engineering (ICCKE), 2014, pp. 741-746, doi: 10.1109/ICCKE.2014.6993452.

[110] Y. Rao, L. Ma, C. Zhao, and Y. Cao, "ANN-Based Multi-scales Prediction of Self-similar Network Traffic," in The Sixth International Symposium on Neural Networks (ISNN 2009), Advances in Intelligent and Soft Computing, Wang H., Shen Y., Huang T., Zeng Z., Ed., Germany: Springer, 2009.

[111] P. Cortez, M. Rio, M. Rocha and P. Sousa, "Internet Traffic Forecasting using Neural Networks," The 2006 IEEE International Joint Conference on Neural Network Proceedings, 2006, pp. 2635-2642, doi: 10.1109/IJCNN.2006.247142.

[112] A. Khotanzad and N. Sadek, "Multi-scale high-speed network traffic prediction using combination of neural networks," Proceedings of the International Joint Conference on Neural Networks, 2003., 2003, pp. 1071-1075 vol.2, doi: 10.1109/IJCNN.2003.1223839.

[113] A. Mozo, B. Ordozgoiti, and S. Gómez-Canaval, "Forecasting short-term data center network traffic load with convolutional neural networks," PLOS one, vol. 13, no. 2, 2018, doi:10.1371/journal.pone.0191939.

[114] T. T. T. Nguyen and G. Armitage," A survey of techniques for internet traffic classification using machine learning," in IEEE Communications Surveys Tutorials, vol. 10, no. 4, pp. 56-76, Fourth Quarter 2008, doi: 10.1109/SURV.2008.080406.

[115] A. K. J. Michael, E. Valla, N. S. Neggatu, and A. W. Moore, "Network traffic classificationvia neural networks," University of Cambridge, Computer Laboratory, Tech. Rep., 2017

[116] L. A. Zadeh, "Fuzzy sets," Information and control, vol. 8, no. 3, pp. 338-353, 1965, doi: 10.1016/S0019-9958(65)90241-X.

[117] K. -B. Sim, K. -S. Byun and F. Harashima, ”Internet-Based Teleoperation of an Intelligent Robot With Optimal Two-Layer Fuzzy Controller,' in IEEE Transactions on Industrial Electronics, vol. 53, no. 4, pp. 1362-1372, June 2006, doi: 10.1109/TIE.2006.878295.

[118] C. Fu-Lai and D. Ji-Cheng , "On multistage fuzzy neural network modeling," in IEEE Transactions on Fuzzy Systems, vol. 8, no. 2, pp. 125-142, April 2000, doi: 10.1109/91.842148.

[119] A. Nasirian and M. A. Khanesar, "Sliding mode fuzzy rule base bilateral teleoperation control of 2DOF SCARA system," 2016 International Conference on Automatic Control and Dynamic Optimization Techniques (ICACDOT), 2016, pp. 7-12, doi: 10.1109/ICACDOT.2016.7877542.

[120] D. Sun, Q. Liao and H. Ren, "Type-2 Fuzzy Modeling and Control for Bilateral Teleoperation System With Dynamic Uncertainties and Time-Varying Delays," in IEEE Transactions on Industrial Electronics, vol. 65, no. 1, pp. 447-459, Jan. 2018, doi: 10.1109/TIE.2017.2719604.

[121] U. Farooq, J. Gu, M. El-Hawary, M. U. Asad and G. Abbas, ”Fuzzy Model Based Bilateral Control 
Design of Nonlinear Tele-Operation System Using Method of State Convergence," in IEEE Access, vol. 4, pp. 4119-4135, 2016, doi: 10.1109/ACCESS.2016.2558524.

[122] M. S. Mahmoud, M. M. Hamdan, and U. A. Baroudi, "Modeling and control of cyber-physical systems subject to cyber attacks: a survey of recent advances and challenges," Neurocomputing, vol. 338, pp. 101-115, 2019, doi: 10.1016/j.neucom.2019.01.099.

[123] M. S. Mahmoud, M. M. Hamdan, and U. A. Baroudi, "Secure control of cyber physical systems subject to stochastic distributed DoS and deception attacks," International Journal of Systems Science, vol. 51, no. 9, pp. 1653-1668, 2020, doi: 10.1080/00207721.2020.1772402.

[124] M. S. Mahmoud and M. M. Hamdan, "Improved control of cyber-physical systems subject to cyber and physical attacks," Cyber-Physical Systems, vol. 5, no. 3, pp. 173-190, 2019, doi: 10.1080/23335777.2019.1631889.

[125] V. S. Dolk, P. Tesi, C. De Persis and W. P. M. H. Heemels, ”Output-based event-triggered control systems under Denial-of-Service attacks," 2015 54th IEEE Conference on Decision and Control (CDC), 2015, pp. 4824-4829, doi: 10.1109/CDC.2015.7402972.

[126] V. S. Dolk, P. Tesi, C. De Persis and W. P. M. H. Heemels, "Event-Triggered Control Systems Under Denial-of-Service Attacks," in IEEE Transactions on Control of Network Systems, vol. 4, no. 1, pp. 93-105, March 2017, doi: 10.1109/TCNS.2016.2613445.

[127] H. Shisheh Foroush and S. Martínez, "On event-triggered control of linear systems under periodic denialof-service jamming attacks," 2012 IEEE 51st IEEE Conference on Decision and Control (CDC), 2012, pp. 2551-2556, doi: 10.1109/CDC.2012.6425868.

[128] C. De Persis and P. Tesi, "Resilient control under denial-of-service," IFAC Proceedings Volumes, vol. 47, no. 3, pp. 134-139, 2014, doi: 10.3182/20140824-6-ZA-1003.02184.

[129] C. De Persis and P. Tesi, "On resilient control of nonlinear systems under Denial-of-Service," 53rd IEEE Conference on Decision and Control, 2014, pp. 5254-5259, doi: 10.1109/CDC.2014.7040210.

[130] C. Yang, W. Yang, and H. Shi, "DoS attack in centralised sensor network against state estimation," IET Control Theory \& Applications, vol. 12, no. 9, pp. 1244-1253, 2018, doi: 10.1049/iet-cta.2017.0819.

[131] S. Amin, X. Litrico, S. Sastry and A. M. Bayen, "Cyber Security of Water SCADA Systems-Part I: Analysis and Experimentation of Stealthy Deception Attacks," in IEEE Transactions on Control Systems Technology, vol. 21, no. 5, pp. 1963-1970, Sept. 2013, doi: 10.1109/TCST.2012.2211873.

[132] L. Ma, Z. Wang and Y. Yuan, "Consensus control for nonlinear multi-agent systems subject to deception attacks," 2016 22nd International Conference on Automation and Computing (ICAC), 2016, pp. 21-26, doi: 10.1109/IConAC.2016.7604888.

[133] X. Huang and J. Dong, "Adaptive optimization deception attack on remote state estimator of aeroengine," 2017 29th Chinese Control And Decision Conference (CCDC), 2017, pp. 5849-5854, doi: 10.1109/CCDC.2017.7978214.

[134] C.-Z. Bai, F. Pasqualetti, and V. Gupta, "Data-injection attacks in stochastic control systems: Detectability and performance tradeoffs," Automatica, vol. 82, pp. 251-260, 2017, doi: 10.1016/j.automatica.2017.04.047.

[135] D. Ding, G. Wei, S. Zhang, Y. Liu, and F. E. Alsaadi, "On scheduling of deception attacks for discretetime networked systems equipped with attack detectors," Neurocomputing, vol. 219, pp. 99-106, 2017, doi: 10.1016/j.neucom.2016.09.009.

[136] H. Yuan and Y. Xia, "Secure filtering for stochastic non-linear systems under multiple missing measurements and deception attacks," IET Control Theory \& Applications, vol. 12, no. 4,pp. 515-523, 2017, doi: 10.1049/iet-cta.2017.0868.

[137] D. Ding, Z. Wang, G. Wei, and F. E. Alsaadi, ”Event-based security control for discrete-time stochastic systems," IET Control Theory \& Applications, vol. 10, no. 15, pp. 1808-1815, 2016, doi: 10.1049/ietcta.2016.0135.

[138] Y. Yuan, P. Zhang, L. Guo, and H. Yang, "Towards quantifying the impact of randomly occurred attacks on a class of networked control systems," Journal of the Franklin Institute, vol. 354, no. 12, pp. 4966-4988, 2017, doi: 10.1016/j.jfranklin.2017.05.016.

[139] T. Rhouma, K. Chabir, and M. N. Abdelkrim, "Resilient Control for Networked Control Systems Subject to Cyber/Physical Attacks," International Journal of Automation and Computing, vol. 15, pp. 345-354, 2018, doi: 10.1007/s11633-017-1059-x.

[140] M. E. Tozal, Yongge Wang, E. Al-Shaer, K. Sarac, B. Thuraisingham and Bei-Tseng Chu, ”On se- 
cure and resilient telesurgery communications over unreliable networks," 2011 IEEE Conference on Computer Communications Workshops (INFOCOM WKSHPS), 2011, pp. 714-719, doi: 10.1109/INFCOMW.2011.5928905.

[141] G. S. Lee and B. Thuraisingham, "Cyberphysical systems security applied to telesurgical robotics," Computer Standards \& Interfaces, vol. 34, no. 1, pp. 225-229, 2012, doi: 10.1016/j.csi.2011.09.001.

[142] T. Bonaci and H. J. Chizeck, "On potential security threats against rescue robotic systems," 2012 IEEE International Symposium on Safety, Security, and Rescue Robotics (SSRR), 2012, pp. 1-2, doi: 10.1109/SSRR.2012.6523908.

[143] T. Bonaci and H. J. Chizeck, "Surgical telerobotics meets information security," 21st Usenix Security Symposium, 2012

[144] T. Bonaci, J. Yan, J. Herron, T. Kohno, and H. J. Chizeck, "Experimental analysis of denial-of-service attacks on teleoperated robotic systems," Proceedings of the ACM/IEEE Sixth International Conference on Cyber-Physical Systems (ICCPS '15), 2015, pp. 11-20, doi: 10.1145/2735960.2735980.

[145] H. Alemzadeh, D. Chen, X. Li, T. Kesavadas, Z. T. Kalbarczyk and R. K. Iyer, "Targeted Attacks on Teleoperated Surgical Robots: Dynamic Model-Based Detection and Mitigation,” 2016 46th Annual IEEE/IFIP International Conference on Dependable Systems and Networks (DSN), 2016, pp. 395-406, doi: 10.1109/DSN.2016.43.

[146] D. Quarta, M. Pogliani, M. Polino, F. Maggi, A. M. Zanchettin and S. Zanero, "An Experimental Security Analysis of an Industrial Robot Controller," 2017 IEEE Symposium on Security and Privacy (SP), 2017, pp. 268-286, doi: 10.1109/SP.2017.20.

[147] H. C. Hu and Y. C. Liu, "Passivity-based control framework for task-space bilateral teleoperation with parametric uncertainty over unreliable networks," ISA Transactions, vol. 70, pp. 187-199, 2017, doi: 10.1016/j.isatra.2017.07.024.

[148] A. Munteanu, R. Muradore, M. Merro and P. Fiorini, ”On cyber-physical attacks in bilateral teleoperation systems: An experimental analysis," 2018 IEEE Industrial Cyber-Physical Systems (ICPS), 2018, pp. 159-166, doi: 10.1109/ICPHYS.2018.8387653.

[149] Y. Dong, N. Gupta and N. Chopra, "On content modification attacks in bilateral teleoperation systems," 2016 American Control Conference (ACC), 2016, pp. 316-321, doi: 10.1109/ACC.2016.7524934.

[150] Y. Dong, N. Gupta and N. Chopra, "False Data Injection Attacks in Bilateral Teleoperation Systems," in IEEE Transactions on Control Systems Technology, vol. 28, no. 3, pp. 1168-1176, May 2020, doi: 10.1109/TCST.2019.2903446. 